\title{
Current noise cross correlation mediated by Majorana bound states
}

\author{
Hai-Feng Lü, ${ }^{1,2}$ Hai-Zhou Lu, ${ }^{2}$ and Shun-Qing Shen ${ }^{2}$ \\ ${ }^{1}$ Department of Applied Physics, University of Electronic Science and Technology of China, Chengdu 610054, China \\ ${ }^{2}$ Department of Physics, The University of Hong Kong, Pokfulam Road, Hong Kong, China
}

(Received 16 March 2014; revised manuscript received 2 October 2014; published 5 November 2014)

\begin{abstract}
We study the transport properties of a quantum dot-Majorana hybrid system, in which each of the paired Majorana bound states is connected to one quantum dot. With the help of the nonequilibrium Green's function method, we obtain an exact solution of the Green's functions and calculate the currents through the quantum dots and nonlocal noise cross correlation between the currents. As a function of dot energy levels $\epsilon_{1}$ and $\epsilon_{2}$, we find that for the symmetric level configuration $\epsilon_{1}=\epsilon_{2}$, the noise cross correlation is negative in the low lead voltage regime, while it becomes positive with the increase of the lead voltages. Due to the particle-hole symmetry, the cross correlation is always positive in the antisymmetric case $\epsilon_{1}=-\epsilon_{2}$. In contrast, the cross correlation of non-Majorana setups is always positive. For comparison, we also perform the diagonalized master equation calculation to check its applicability. It is found that the diagonalized master equations work well in most regimes of system parameters. Nevertheless, it shows an obvious deviation from the exact solution by the nonequilibrium Green's function method when all eigenenergies of the dot-Majorana hybrid system and simultaneously the energy intervals are comparable to the dot-lead coupling strength.
\end{abstract}

DOI: 10.1103/PhysRevB.90.195404

PACS number(s): 03.75.Lm, 72.10.-d, 74.78.Na, 73.21.La

\section{INTRODUCTION}

Majorana fermions, defined as fermions equivalent to own antiparticles, have been hunted for by high energy physicists for a long time [1-3]. In recent years, the search of Majorana fermions has been shifted to solid-state systems, such as in the fractional quantum Hall system and $p$-wave superconductors [2-8]. In particular, the Majorana bound states (MBSs) are predicted to appear at two ends of a semiconductor nanowire, in the proximity of an $s$-wave superconductor and under a proper magnetic field [9-17]. The signatures for possible formation of a spatially separated pair of MBSs were reported in several experiments [18-22]. Two well-separated MBSs can define a nonlocal fermion level and its occupation encodes a qubit [5-7]. This nonlocal topological qubit is immune to local perturbation and thus has potential application in quantum information. However, for the same reason, it is a great challenge to coherently transfer and to read out the quantum information of the topological states $[7,17,23]$. It has been suggested that the MBS-quantum dot hybrid system might be one of the solutions to the problem $[7,23,24]$. Up to now, various MBS-dot hybrid devices [23-35] have been proposed to detect the existence of MBS [26,28,29], to modulate nonlocal correlation [30-34], to estimate lifetime [27], and to remove the effect of disorder [35]. Therefore, it is important to investigate the transport properties of the MBS-dot hybrid systems.

One of the fascinating properties of MBSs is that MBSs could induce nonlocal current cross correlation when they are coupled to mesoscopic circuits [36-41]. It has been shown that a positive cross correlation could be induced when MBSs couple to electron reservoirs directly [36-38]. In our previous work, we proposed a device to modulate the Majoranafermion-mediated cross correlation of the currents flowing through two quantum dots located between the MBSs and reservoirs [30]. Subsequently, the time-dependent evaluation of electron population and other related transport properties were investigated by the scattering matrix theory $[31,32]$ and the master equation approach $[33,34]$, respectively.
In this paper, we systematically investigate the nonlocal transport properties of the quantum dot-Majorana hybrid systems (as shown in Fig. 1) by means of both the nonequilibrium Green's function (NEGF) method and diagonalized master equation (DME) approach, respectively. The currents through the quantum dots and nonlocal cross correlation between the currents are investigated as functions of lead voltage, dot energy levels, Majorana energy splitting, dot-Majorana coupling, and temperature. In the weak dot-lead coupling regime, we obtain the analytical expressions of the currents and their noise correlation. The sign distribution of the cross correlation is analyzed (summarized in Table I), which shows a distinct four-region feature from pure quantum-dot or superconducting circuits (see Figs. 4 and 5 for comparison). Furthermore, in the noninteracting case, the NEGF method gives the exact solution, offering a benchmark to check the applicability of DME. The paper is organized as follows. In Sec. II, the model Hamiltonian of the dot-Majorana hybrid device, as well as the current and noise cross correlation formulas in terms of NEGF and DME, are introduced. The details of derivation are given in the Appendices. In Sec. III, the numerical results for the current and noise cross correlation are presented. In Sec. IV, the applicability of the DME approach is discussed by comparing with the exact solution by the NEGF method. Finally, a summary is given in Sec. V.

\section{MODEL AND FORMULISM}

\section{A. Model}

The schematic of the setup is illustrated in Fig. 1. Two quantum dots are connected to a one-dimensional semiconductor nanowire with strong Rashba spin-orbit coupling [25,30]. Under a proper magnetic field, the semiconductor nanowire resembles a topological superconductor when it is adjacent to an $s$-wave superconductor due to the proximity effect [9-17]. At the ends of the wire, a pair of MBSs emerges. Each dot is connected to one of the MBSs and one normal metal lead. 


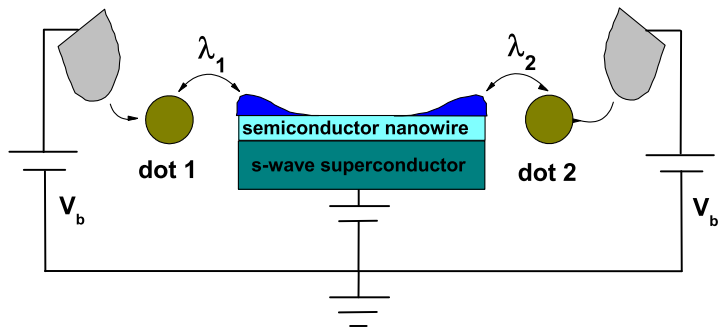

FIG. 1. (Color online) Schematic of a quantum dot-Majorana hybrid system. Two quantum dots are coupled to two ends of a semiconductor nanowire with strong spin-orbit interaction. The nanowire is in contact with an $s$-wave superconductor. In a large enough Zeeman field, the nanowire is driven into the topological superconducting phase and a pair of Majorana bound states appears at its ends. Each dot is connected with a normal metal lead.

Experimentally, the magnetic field is up to several Tesla, which can produce a Zeeman splitting in each dot as well such that the spin degree of freedom of electrons can be suppressed. Consequently the model Hamiltonian is expressed as

$$
H=H_{0}+H_{L}+H_{T} .
$$

The quantum dot-Majorana hybrid part is described by

$$
\begin{aligned}
H_{0}= & \sum_{j=1,2} \epsilon_{j} d_{j}^{\dagger} d_{j}+\frac{i}{2} \epsilon_{M} \eta_{a} \eta_{b}+\lambda_{1}\left(d_{1}^{\dagger}-d_{1}\right) \eta_{a} \\
& +i \lambda_{2}\left(d_{2}^{\dagger}+d_{2}\right) \eta_{b},
\end{aligned}
$$

where $\epsilon_{j}$ is the energy level in quantum dot $j(j=1,2)$ and $d_{j}\left(d_{j}^{\dagger}\right)$ is the annihilation (creation) operator of electron. The quantum dots $j$ are coupled to the MBSs with the strength $\lambda_{j} . \epsilon_{M j}$ is the coupling strength between two MBSs $\eta_{j a}\left(=\eta_{j a}^{\dagger}\right)$ and $\eta_{j b}\left(=\eta_{j b}^{\dagger}\right)$. The electron reservoirs and their coupling to the dots are described by the Hamiltonian,

$$
\begin{aligned}
& H_{L}=\sum_{j k} \epsilon_{j, k} c_{j k}^{\dagger} c_{j k}, \\
& H_{T}=\sum_{j, k}\left(t_{j} c_{j k}^{\dagger} d_{j}+\text { H.c. }\right),
\end{aligned}
$$

where $\epsilon_{j, k}$ is the electron energy in the lead $j$ and $t_{j}$ is the tunneling amplitude.

For convenience, we can switch from the Majorana fermion representation to the regular fermion representation by defining $\eta_{a}=f+f^{\dagger}, \eta_{b}=i\left(f^{\dagger}-f\right)$ with $f^{\dagger} f-f f^{\dagger}=1$

TABLE I. Sign distribution of noise correlation $S_{12}$ with the increase of lead voltage $V_{0}$ in the weak dot-lead coupling regime. For simplicity, we consider the case of symmetric Majorana-dot and dot-lead coupling strength, i.e., $\Gamma_{1}=\Gamma_{2}$ and $\lambda_{1}=\lambda_{2}$.

\begin{tabular}{lccc}
\hline \hline Cases & Small $V_{0}$ & $\Rightarrow$ & Large $V_{0}$ \\
\hline$\epsilon_{1}>0, \epsilon_{2}>0$ & - & - & + \\
$\epsilon_{1}>0, \epsilon_{2}<0$ & + & + & + \\
$\epsilon_{1}<0, \epsilon_{2}<0$ & - & + & + \\
$\epsilon_{1}<0, \epsilon_{2}>0$ & + & + & + \\
\hline \hline
\end{tabular}

$[25,26,30]$. In the new representation, the Hamiltonian in the central region becomes [30]

$$
\begin{aligned}
H_{0}= & \sum_{j=1,2} \epsilon_{j} d_{j}^{\dagger} d_{j}+\epsilon_{M}\left(f^{\dagger} f-\frac{1}{2}\right) \\
& +\left[\lambda_{1}\left(f^{\dagger} d_{1}+f d_{1}\right)+\lambda_{2}\left(f^{\dagger} d_{2}-f d_{2}\right)+\text { H.c. }\right]
\end{aligned}
$$

\section{B. Current and noise cross correlation}

The operator of tunneling current from the lead $\alpha$ to the central region can be given by

$$
\begin{aligned}
\mathbf{I}_{\alpha}(t) & \equiv-e \frac{d \hat{N}_{\alpha}}{d t}=\frac{i e}{\hbar}\left[\hat{N}_{\alpha}, \hat{H}\right] \\
& =\frac{i e}{\hbar} t_{\alpha} \sum_{k}\left(c_{\alpha k}^{\dagger} d_{\alpha}-d_{\alpha}^{\dagger} c_{\alpha k}\right) .
\end{aligned}
$$

The current noise correlations are defined as

$$
S_{12}\left(t-t^{\prime}\right)=\left\langle I_{1}(t) I_{2}\left(t^{\prime}\right)+I_{2}\left(t^{\prime}\right) I_{1}(t)\right\rangle-2\left\langle I_{1}\right\rangle\left\langle I_{2}\right\rangle .
$$

$S_{12}$ is referred to as the noise cross correlation between the currents flowing through dot 1 and dot 2 .

The quantum transport of many-body systems can be obtained by several techniques. In the following subsections, we will calculate the currents and their noise cross correlations using both the standard Keldysh NEGF method [42-44] and the DME approach [45-50].

\section{Nonequilibrium Green's function method}

Following Ref. [44], we define the matrix of lesser Green's function $\mathbf{G}^{<}$in the Nambu space spanned by the spinor $\psi=\left(d_{1}, d_{2}, f, c_{1}, c_{2}, d_{1}^{\dagger}, d_{2}^{\dagger}, f^{\dagger}, c_{1}^{\dagger}, c_{2}^{\dagger}\right)^{T}$, where $c_{i}$ is the electron annihilation operator in lead $i$ and

$$
\mathbf{G}^{<}\left(t-t^{\prime}\right)=\left\langle\left\langle\psi(t) \mid \psi^{\dagger}\left(t^{\prime}\right)\right\rangle\right\rangle^{<}=i\left\langle\psi^{\dagger}\left(t^{\prime}\right) \psi(t)\right\rangle .
$$

In the frequency space,

$$
\mathbf{G}_{\omega}^{<}=\int d t \mathbf{G}^{<}(t) e^{i \omega t} .
$$

In this representation, the currents are given by

$$
I_{\alpha}=\operatorname{Tr}\left[\hat{\mathbf{I}}_{\alpha} \mathbf{G}^{<}\right],
$$

and the noise spectrum $S_{12}(\omega)$ is given by

$$
S_{12}(\omega)=\int d \omega^{\prime} \operatorname{Tr}\left[\hat{\mathbf{I}}_{1} \mathbf{G}_{\omega^{\prime}}^{<} \hat{\mathbf{I}}_{2} \mathbf{G}_{\omega^{\prime}+\omega}^{>}+\hat{\mathbf{I}}_{2} \mathbf{G}_{\omega^{\prime}}^{>} \hat{\mathbf{I}}_{1} \mathbf{G}_{\omega^{\prime}+\omega}^{<}\right] .
$$

The matrices of the current operators are given by

$$
\begin{aligned}
& \mathbf{I}_{1}=\frac{e}{2 \hbar} t_{1}\left(\begin{array}{ll}
1 & 0 \\
0 & 1
\end{array}\right) \otimes\left(\begin{array}{rrrrr}
0 & 0 & 0 & -1 & 0 \\
0 & 0 & 0 & 0 & 0 \\
0 & 0 & 0 & 0 & 0 \\
1 & 0 & 0 & 0 & 0 \\
0 & 0 & 0 & 0 & 0
\end{array}\right), \\
& \mathbf{I}_{2}=\frac{e}{2 \hbar} t_{2}\left(\begin{array}{ll}
1 & 0 \\
0 & 1
\end{array}\right) \otimes\left(\begin{array}{ccccc}
0 & 0 & 0 & 0 & 0 \\
0 & 0 & 0 & 0 & -1 \\
0 & 0 & 0 & 0 & 0 \\
0 & 0 & 0 & 0 & 0 \\
0 & 1 & 0 & 0 & 0
\end{array}\right) .
\end{aligned}
$$


To find the lesser Green's function $\mathbf{G}^{<}$, we need to calculate the retarded Green's function $\mathbf{G}^{r}$. As there is no four-operator interaction term in the Hamiltonian, the Green's function can be solved analytically. Performing the standard equation of motion procedure for the central region, the retarded Green's function $\mathbf{G}^{r}$ can be found and written in terms of the Dyson equation $\mathbf{G}^{r}=\mathbf{g}^{r}+\mathbf{g}^{r} \Sigma^{r} \mathbf{G}^{r}$,

$$
\mathbf{G}^{\mathbf{r}}=\left(1-\mathbf{g}^{r} \Sigma^{r}\right)^{-1} \mathbf{g}^{r} .
$$

Here $\mathbf{g}^{r}$ is the bare Green's function of the central region when isolated from the leads (i.e., when $t_{1}=t_{2}=0$ ),

$$
\begin{aligned}
\mathbf{g}^{r}(\omega)^{-1}= & \left(\begin{array}{ll}
1 & 0 \\
0 & 1
\end{array}\right) \otimes\left(\begin{array}{cccccc}
\omega & 0 & 0 & 0 & 0 \\
0 & \omega & 0 & 0 & 0 \\
0 & 0 & \omega & 0 & 0 \\
0 & 0 & 0 & i / \pi \rho & 0 \\
0 & 0 & 0 & 0 & i / \pi \rho
\end{array}\right) \\
& -\left(\begin{array}{rr}
1 & 0 \\
0 & -1
\end{array}\right) \otimes\left(\begin{array}{ccccc}
\epsilon_{1} & 0 & \lambda_{1} & 0 & 0 \\
0 & \epsilon_{2} & \lambda_{2} & 0 & 0 \\
\lambda_{1} & \lambda_{2} & \epsilon_{M} & 0 & 0 \\
0 & 0 & 0 & 0 & 0 \\
0 & 0 & 0 & 0 & 0
\end{array}\right) \\
& +\left(\begin{array}{cc}
0 & 1 \\
-1 & 0
\end{array}\right) \otimes\left(\begin{array}{cccccc}
0 & 0 & -\lambda_{1} & 0 & 0 \\
0 & 0 & \lambda_{2} & 0 & 0 \\
\lambda_{1} & -\lambda_{2} & 0 & 0 & 0 \\
0 & 0 & 0 & 0 & 0 \\
0 & 0 & 0 & 0 & 0
\end{array}\right),
\end{aligned}
$$

and the self-energy $\Sigma^{r}$ has the form

$$
\Sigma^{r}=\left(\begin{array}{rr}
1 & 0 \\
0 & -1
\end{array}\right) \otimes\left(\begin{array}{ccccc}
0 & 0 & 0 & t_{1} & 0 \\
0 & 0 & 0 & 0 & t_{2} \\
0 & 0 & 0 & 0 & 0 \\
t_{1} & 0 & 0 & 0 & 0 \\
0 & t_{2} & 0 & 0 & 0
\end{array}\right)
$$

Using the relationship $\mathbf{G}^{r}=\left(\mathbf{G}^{a}\right)^{\dagger}$, the advanced Green's function can be found. It is straightforward to have the lesser Green's function from the standard Keldysh equation,

$$
\begin{aligned}
\mathbf{G}^{<} & =\left(1+\mathbf{G}^{r} \Sigma^{r}\right) \mathbf{g}^{<}\left(1+\Sigma^{a} \mathbf{G}^{a}\right)+\mathbf{G}^{r} \Sigma^{<} \mathbf{G}^{a} \\
& =\mathbf{G}^{r} \mathbf{g}^{r-1} \mathbf{g}^{<} \mathbf{g}^{a-1} \mathbf{G}^{a}+\mathbf{G}^{r} \Sigma^{<} \mathbf{G}^{a} .
\end{aligned}
$$

In the present case, $\Sigma^{<}=0$ and

$$
\mathbf{g}^{r-1} \mathbf{g}^{<} \mathbf{g}^{a-1}=\left(\begin{array}{cc}
\Lambda_{e} & 0 \\
0 & \Lambda_{h}
\end{array}\right)
$$

with

$$
\Lambda_{e(h)}=\left(\begin{array}{ccccc}
0 & 0 & 0 & 0 & 0 \\
0 & 0 & 0 & 0 & 0 \\
0 & 0 & 0 & 0 & 0 \\
0 & 0 & 0 & \frac{2 i}{\pi \rho} f\left(\omega \mp \mu_{1}\right) & 0 \\
0 & 0 & 0 & 0 & \frac{2 i}{\pi \rho} f\left(\omega \mp \mu_{2}\right)
\end{array}\right),
$$

where $f(\omega)=\left[1+e^{\omega / k_{B} T}\right]^{-1}$ is the Fermi-Dirac distribution function and $k_{B} T$ is the temperature.

\section{Diagonalized master equations}

The electronic transport through this system in a sequential tunneling regime can also be described by the quantum rate equations for dynamical evolution of the density matrix elements [46-50]. In this subsection we present the formula of the currents and their cross correlation in the diagonalized representation [49]. In comparison with the NEGF method and the regular master equation approach, the DME approach is convenient in the calculation of many interacting and strongly coherent energy levels.

In the dot-Majorana hybrid part, there are eight states in the particle-number representation $\left|n_{1} n_{2} n_{M}\right\rangle$, where $n_{i}=0,1$ is the occupation numbers in dot $i$ and the MBS. By choosing the basis $\{|000\rangle,|101\rangle,|011\rangle,|110\rangle,|001\rangle,|100\rangle,|010\rangle,|111\rangle\}$, the eigenequation for the dot-Majorana Hamiltonian is given by

$$
\left(\begin{array}{cc}
\mathbf{M}^{e} & 0 \\
0 & \mathbf{M}^{o}
\end{array}\right) \Psi_{i}=E_{i} \Psi_{i}
$$

where

$$
\begin{aligned}
\mathbf{M}^{e} & =\left(\begin{array}{cccc}
-\frac{\epsilon_{M}}{2} & \lambda_{1} & -\lambda_{2} & 0 \\
\lambda_{1} & \epsilon_{1}+\frac{\epsilon_{M}}{2} & 0 & \lambda_{2} \\
-\lambda_{2} & 0 & \epsilon_{2}+\frac{\epsilon_{M}}{2} & -\lambda_{1} \\
0 & \lambda_{2} & -\lambda_{1} & \epsilon_{1}+\epsilon_{2}-\frac{\epsilon_{M}}{2}
\end{array}\right), \\
\mathbf{M}^{o} & =\left(\begin{array}{cccc}
\frac{\epsilon_{M}}{2} & \lambda_{1} & \lambda_{2} & 0 \\
\lambda_{1} & \epsilon_{1}-\frac{\epsilon_{M}}{2} & 0 & -\lambda_{2} \\
\lambda_{2} & 0 & \epsilon_{2}-\frac{\epsilon_{M}}{2} & -\lambda_{1} \\
0 & -\lambda_{2} & -\lambda_{1} & \epsilon_{1}+\epsilon_{2}+\frac{\epsilon_{M}}{2}
\end{array}\right) .
\end{aligned}
$$

As the matrix is block diagonalized, we denote the eigenstates in terms of $\Psi_{l}^{o(e)}=\left(a_{l}^{o(e)}, b_{l}^{o(e)}, c_{l}^{o(e)}, d_{l}^{o(e)}\right)^{\mathbf{T}}$ for

$$
\mathbf{M}^{o(e)} \Psi_{l}^{o(e)}=E_{l}^{o(e)} \Psi_{l}^{o(e)} .
$$

In the DME approach the density matrix $\rho_{D}(t)=\left|\Psi_{i}\right\rangle\left\langle\Psi_{i^{\prime}}\right|$ has only diagonal terms that represent the populations in the states $\Psi_{i}^{o(e)}$, and its time evaluation is governed by the rate equation

$$
\frac{d}{d t} \rho_{D}(t)=\mathbf{W} \rho_{D}(t),
$$

where the elements of the rate matrix are given by $[30,49]$

$$
\begin{aligned}
W_{k^{\prime} k}= & \sum_{i} \Gamma_{i}\left[f\left(\Delta_{k^{\prime} k}+\mu_{i}\right)\left|\left\langle\beta_{k^{\prime}}\left|d_{i}\right| \beta_{k}\right\rangle\right|^{2}\right. \\
& \left.+f\left(\Delta_{k^{\prime} k}-\mu_{i}\right)\left|\left\langle\beta_{k^{\prime}}\left|d_{i}^{\dagger}\right| \beta_{k}\right\rangle\right|^{2}\right]
\end{aligned}
$$

for $k \neq k^{\prime}$ and

$$
W_{k k}=-\sum_{k^{\prime} \neq k}^{N} W_{k^{\prime} k} .
$$

Here $\mu_{i}$ is the chemical potential in lead $i$, and $\Delta_{k^{\prime} k}$ is the Bohr frequency of the transition from $\left|\beta_{k}\right\rangle$ to $\left|\beta_{k^{\prime}}\right\rangle$. In the wide-band limit approximation, the coupling between dot $i$ and its lead is denoted by $\Gamma_{i}=2 \pi\left|t_{i}\right|^{2} \rho_{i}$ with $\rho_{i}$ the spinless density of states near the Fermi surface of lead $i$. 
The steady-state current $I_{i}$ is given by

$$
I_{i}=e \sum_{k}\left[\hat{\Gamma}^{i} \rho_{D}^{(0)}\right]_{k}
$$

where $\hat{\Gamma}^{i}$ is the matrix of the current operator and its element is given by

$$
\begin{aligned}
\hat{\Gamma}_{k^{\prime} k}^{i}= & \Gamma_{i}\left[f\left(\Delta_{k^{\prime} k}+\mu_{i}\right)\left|\left\langle\beta_{k^{\prime}}\left|d_{i}\right| \beta_{k}\right\rangle\right|^{2}\right. \\
& \left.-f\left(\Delta_{k^{\prime} k}-\mu_{i}\right)\left|\left\langle\beta_{k^{\prime}}\left|d_{i}^{\dagger}\right| \beta_{k}\right\rangle\right|^{2}\right] .
\end{aligned}
$$

The first term of $\hat{\Gamma}_{k^{\prime} k}^{i}$ is the tunneling current flowing into the lead, and the second term is the tunneling current flowing from the lead to dot.

We are interested in the cross correlation induced by the MBS between the currents through the quantum dots. The noise power spectra can be expressed as the Fourier transform of the current-current correlation function

$$
S_{I_{i} I_{j}}(\omega)=2\left\langle I_{i}(t) I_{j}(0)\right\rangle_{\omega}-2\left\langle I_{i}\right\rangle_{\omega}\left\langle I_{j}\right\rangle_{\omega},
$$

where $I_{i}$ is the current in dot $i$ and $t$ is the time. Furthermore, the current-current correlation function can be expressed in the $\rho$ representation as follows:

$$
\begin{aligned}
\left\langle I_{i}(t) I_{j}(0)\right\rangle= & \theta(t) \sum_{k}\left[\hat{\Gamma}^{i} \hat{T}(t) \hat{\Gamma}^{j} \rho^{(0)}\right]_{k} \\
& +\theta(-t) \sum_{k}\left[\hat{\Gamma}^{j} \hat{T}(-t) \hat{\Gamma}^{i} \rho^{(0)}\right]_{k}
\end{aligned}
$$

with $\hat{T}(t)=\exp [\mathbf{W} t]$ the propagator governing the time evaluation of the density matrix element $\rho_{k}(t)$. Finally, the current-current correlation in the $\omega$ space becomes

$$
\left\langle I_{i}(t) I_{j}(0)\right\rangle_{\omega}=\sum_{k}\left[\hat{\Gamma}^{i} \hat{T}(\omega) \hat{\Gamma}^{j} \rho^{(0)}+\hat{\Gamma}^{j} \hat{T}(-\omega) \hat{\Gamma}^{i} \rho^{(0)}\right]_{k},
$$

where $\hat{T}( \pm \omega)=(\mp i \omega \hat{I}-\mathbf{W})^{-1}$.

\section{CURRENTS AND NOISE CROSS CORRELATION}

In this section, we will calculate the current and zerofrequency cross correlation $S_{12}$ as functions of the lead voltage, Majorana energy splitting, dot energy levels, and temperature. The results by both the NEGF method and the DME approach will be presented simultaneously for comparison. In most calculations, we use the same dot-lead tunneling rate and dot-Majorana coupling strength for both dots, i.e., $\Gamma_{i}=\Gamma_{0}$ and $\lambda_{i}=\lambda_{0}$, while the effects of asymmetric dot-lead and dot-Majorana coupling are discussed in Sec. III E. All energies and frequencies are measured in units of $\Gamma_{0}$. The voltages $V_{0}=\mu_{1}=\mu_{2}$ are symmetrically applied to both leads.

\section{A. Tuning lead voltage}

In Fig. 2 we show the charge current $I_{1}$ and cross correlation $S_{12}$ as functions of the voltage $V_{0}$ for different values of the Majorana energy splitting $\epsilon_{M}$. As shown in Fig. 2, the currents and cross correlations obtained from the two methods agree very well with each other. At low temperatures, the I-V curve exhibits the standard staircase behavior, ascending in steps and rising to a higher plateau every time the chemical potential of
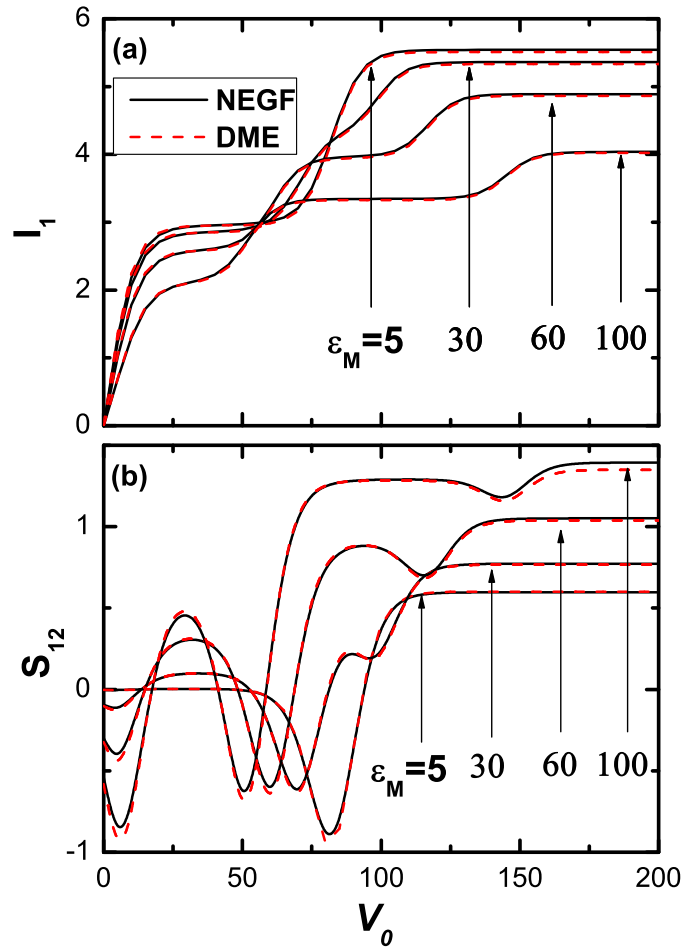

FIG. 2. (Color online) The current $I_{1}$ through dot 1 (top) and zero-frequency cross correlation $S_{12}$ (bottom) as functions of the voltage $V_{0}$ in the leads, for different Majorana energy splitting $\epsilon_{M}=5,30,60$, and 100, respectively. The results obtained from the nonequilibrium Green's function (NEGF) method and the diagonalized master equation (DME) approach are compared. Other parameters: $k_{B} T=5, \lambda_{0}=40, \epsilon_{1}=20$, and $\epsilon_{2}=25$, where the dot-lead coupling strength $\Gamma_{0}$ is taken as the energy unit.

the leads $\mu_{i}$ crosses a higher energy level of the central region. For low voltage $\mu_{i}$, electrons in the leads do not have enough energy to tunnel into the dots, and the currents are contributed by thermal excitations. As the chemical potential of the lead exceeds the eigenenergies one by one, the transport channels are opened correspondingly. Figure 2 considers the case that both dot energy levels are higher than the middle position of the superconducting gap. At a relatively low voltage, the cross correlation favors a negative correlation, corresponding to the competitive relationship between two tunneling paths. With the increase of voltage, the cross correlation shows a sign reversal and becomes positive, indicating that the correlation between two channels becomes cooperative.

\section{B. Effect of Majorana energy splitting}

The Majorana energy splitting $\epsilon_{M}$ between two MBSs is a key parameter to generate nonlocal cross correlation, and the dependence of the cross correlation on $\epsilon_{M}$ is shown in Fig. 3. It has been demonstrated theoretically [51] and experimentally [22] that the splitting $\epsilon_{M}$ has an oscillatory dependence on the Zeeman field and the chemical potential in the nanowire, and exponentially decays as a function of the wire length. For a nanowire with its length $\sim 1 \mu \mathrm{m}$, the typical energy range of $\epsilon_{M}$ is about $0-50 \mu \mathrm{eV}$ [51]. In the limit of $\epsilon_{M}=0$, there is no overlap between the wave functions of 

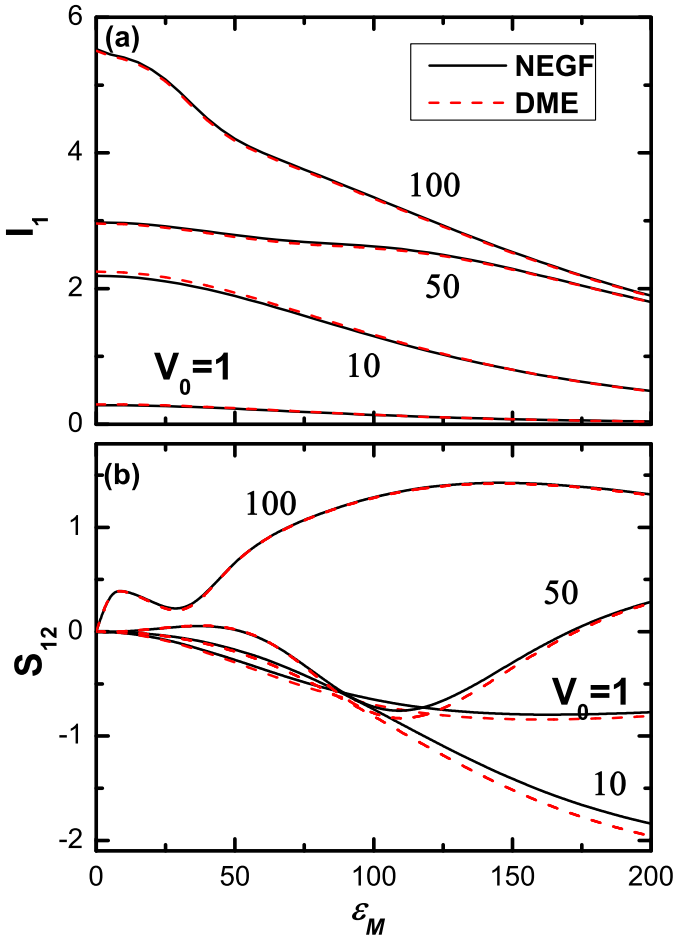

FIG. 3. (Color online) The current $I_{1}$ (top) and zero-frequency cross correlation $S_{12}$ (bottom) as functions of $\epsilon_{M}$ for different lead voltages $V_{0}=1,10,50$, and 100 , respectively. Other parameters are $k_{B} T=5, \lambda_{0}=40, \epsilon_{1}=20$, and $\epsilon_{2}=25$.

two MBSs. The interaction between dot $i$ and MBS on one side changes the parity of the MBS, but does not affect the dot-Majorana interaction on the other side. In this case, there is no communication between two MBSs, corresponding to a zero $S_{12}$. It has been pointed out that when $\epsilon_{M}$ approaches zero, the cross correlation $S_{12}$ is proportional to $\epsilon_{M}^{2}$ and thus vanishes at the point $\epsilon_{M}=0$ [31]. We show that the results are confirmed by both NEGF and DME calculations. With the increase of $\epsilon_{M}$, the current $I_{1}$ is suppressed to zero gradually due to the detuning of the dot-Majorana interaction. At relatively low voltage $V_{0}$, the lowest eigenenergy level will be shifted out of the transport window if $\epsilon_{M}$ is large enough. Correspondingly, the cross correlation $S_{12}$ is governed by the thermal noise for $\epsilon_{M} \gg V_{0}$.

\section{Tuning dot energy levels}

One of the roles of the quantum dots is to control the nonlocal cross correlation by tuning the dot energy levels, for potential applications in the Majorana-based topological quantum information [30]. Experimentally, typical values are a dot-lead coupling $\Gamma_{i}$ in the order of $1 \mu \mathrm{eV}$, which is much weaker than other system parameters. In the weak dot-lead coupling limit, we find the explicit expressions of the current and noise cross correlation (the details can be found in Appendix C). For the symmetric level configuration $\left(\epsilon_{0}=\right.$ $\left.\epsilon_{1}=\epsilon_{2}\right)$, and in the limit of large lead voltage $V_{0}$, we have

$$
S_{12} \approx \int d \omega \frac{16 \epsilon_{M}^{2} \lambda_{0}^{4} \Gamma_{0}^{2}\left(\epsilon_{0}^{2}-\omega^{2}\right)^{2}}{\left[A_{\omega}+B_{\omega}^{2} / A_{\omega}\right]^{2}},
$$

where $A_{\omega}$ and $B_{\omega}$ are defined as

$$
\begin{aligned}
& A_{\omega}=\left(\omega^{2}-\epsilon_{M}^{2}\right)\left(\epsilon_{0}^{2}-\omega^{2}\right)^{2}+8 \lambda_{0}^{2} \omega^{2}\left(2 \lambda_{0}^{2}+\epsilon_{0}^{2}-\omega^{2}\right), \\
& B_{\omega}=2 \Gamma_{0} \omega\left[\left(\omega^{2}-4 \lambda_{0}^{2}-\epsilon_{0}^{2}\right)\left(\omega^{2}-2 \lambda_{0}^{2}-\epsilon_{M}^{2}\right)-4 \lambda_{0}^{2} \epsilon_{M}^{2}\right] .
\end{aligned}
$$

It can be seen that $S_{12}$ is always positive in this case. In the small $V_{0}$ limit,

$$
S_{12} \approx \int d \omega \frac{16 \epsilon_{M}^{2} \lambda_{0}^{4} \Gamma_{0}^{2}\left[\left(4 \epsilon_{0} \omega\right)^{2}\left[\left(f_{\omega}-1\right) f_{\omega}\right]\right]}{\left[A_{\omega}+B_{\omega}^{2} / A_{\omega}\right]^{2}},
$$

where the Fermi function $f_{\omega} \in[0,1]$, and $S_{12}$ is always negative. Moreover, one can check in Eq. (C2) that $S_{12}\left(\epsilon_{0}\right)<$ $S_{12}\left(-\epsilon_{0}\right)$ for $\epsilon_{0}>0$ and any $V_{0}$. Therefore, with the increase of the lead voltage, $S_{12}$ in the regime $\epsilon_{0}<0$ experiences a sign reversal from negative to positive. As $V_{0}$ increases further, the sign reversal of $S_{12}$ then occurs in the regime $\epsilon_{0}>0$.

For the antisymmetric level configuration $\left(\epsilon_{0}=\epsilon_{1}=-\epsilon_{2}\right)$, in the large lead voltage limit,

$$
S_{12} \approx \int d \omega \frac{16 \epsilon_{M}^{2} \lambda_{0}^{4} \Gamma_{0}^{2}\left[\left(\epsilon_{0}^{2}-\omega^{2}\right)^{2}+8 \epsilon_{0}^{2} \omega^{2}\right]}{\left[A_{\omega}+B_{\omega}^{2} / A_{\omega}\right]^{2}},
$$

and in the small voltage limit

$$
S_{12} \approx \int d \omega \frac{16 \epsilon_{M}^{2} \lambda_{0}^{4} \Gamma_{0}^{2}\left[8 \epsilon_{0}^{2} \omega^{2}\left(f_{\omega}^{+}-f_{\omega}^{-}\right)^{2}\right]}{\left[A_{\omega}+B_{\omega}^{2} / A_{\omega}\right]^{2}} .
$$

Therefore, $S_{12}$ is always positive in the antisymmetric level configuration. Different from the symmetric case, $S_{12}$ is an even function of $\epsilon_{0}$ for the antisymmetric level configuration, due to the particle-hole symmetry.

From the above discussion, we could conclude the sign of $S_{12}$ as a function of dot energy levels in the limit of weak dot-lead coupling, as presented in Table I. In Fig. 4 we further demonstrate the cross correlation $S_{12}$ obtained from the NEGF method as functions of the dot energy levels $\epsilon_{i}$ for different voltages $V_{0}$, which is consistent with the analytical results. For the small lead voltage comparable to the dot-lead coupling strength, a four peaked cloverlike pattern of noise cross correlation is illustrated when modulating the dot energy levels. In the previous experiments, such a four peaked cloverlike pattern is also demonstrated in a capacitively-coupled double dot device, where the sign of the cross correlation is independent on the lead voltage [52,53]. For the present Majorana-dot hybrid device, the lead voltage determines which eigenlevel participates in the transport. With the increase of $V_{0}, S_{12}$ in the region of $\epsilon_{1,2}<0$ becomes positive firstly and is much weaker than other regions. For large enough $V_{0}$, the transport channels through all eigenlevels are open and their interaction leads to the positive $S_{12}$ in all regions. In this case, the cross correlation $S_{12}$ in the region of antisymmetric level configuration $\epsilon_{1}=$ $-\epsilon_{2}$ is much stronger than that for the symmetric case.

The signature of Majorana fermions in the cross correlation is different from those of non-Majorana setups. In our setup, the key feature of the cross correlation is the four peaked cloverlike pattern as a function of the dot levels (as illustrated in Fig. 4), due to crossed Andreev reflections mediated by the MBSs. In contrast, the sign distribution is absent in the experiments of other normal superconductor-quantum dot 


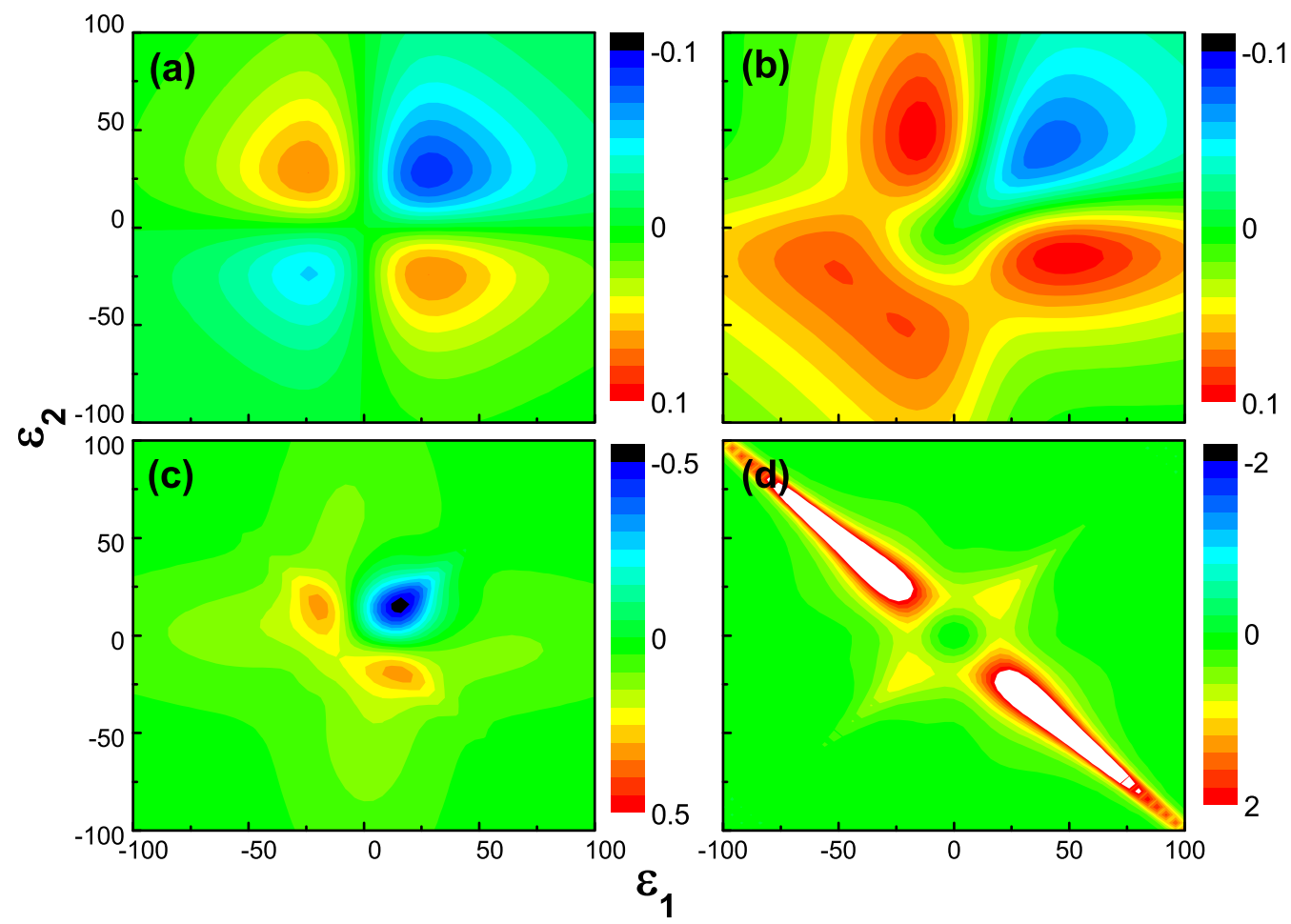

FIG. 4. (Color online) The zero-frequency cross correlation $S_{12}$ obtained from the NEGF method as functions of dot energy levels $\epsilon_{1}$ and $\epsilon_{2}$ for different voltages (a) $V_{0}=1$, (b) 10 , (c) 30, and (d) 200, respectively. Other parameters are $k_{B} T=5, \lambda_{0}=20$, and $\epsilon_{M}=8$.

hybrid systems [54-57]. Also, a setup with a normal $s$-wave superconductor was theoretically studied, but there the cross correlation was not addressed [58]. We calculate the cross correlation of this non-Majorana setup for comparison (the details can be found in Appendix D). As shown in Fig. 5, the cloverlike cross correlation distribution in Fig. 4 is absent in the non-Majorana setup, where the cross correlation is always positive and the peaks appear at $\epsilon_{1}=-\epsilon_{2}= \pm V_{0}$. The comparison of Figs. 4 and 5 gives a distinguishable signature of the MBSs in the cross correlation spectrum.
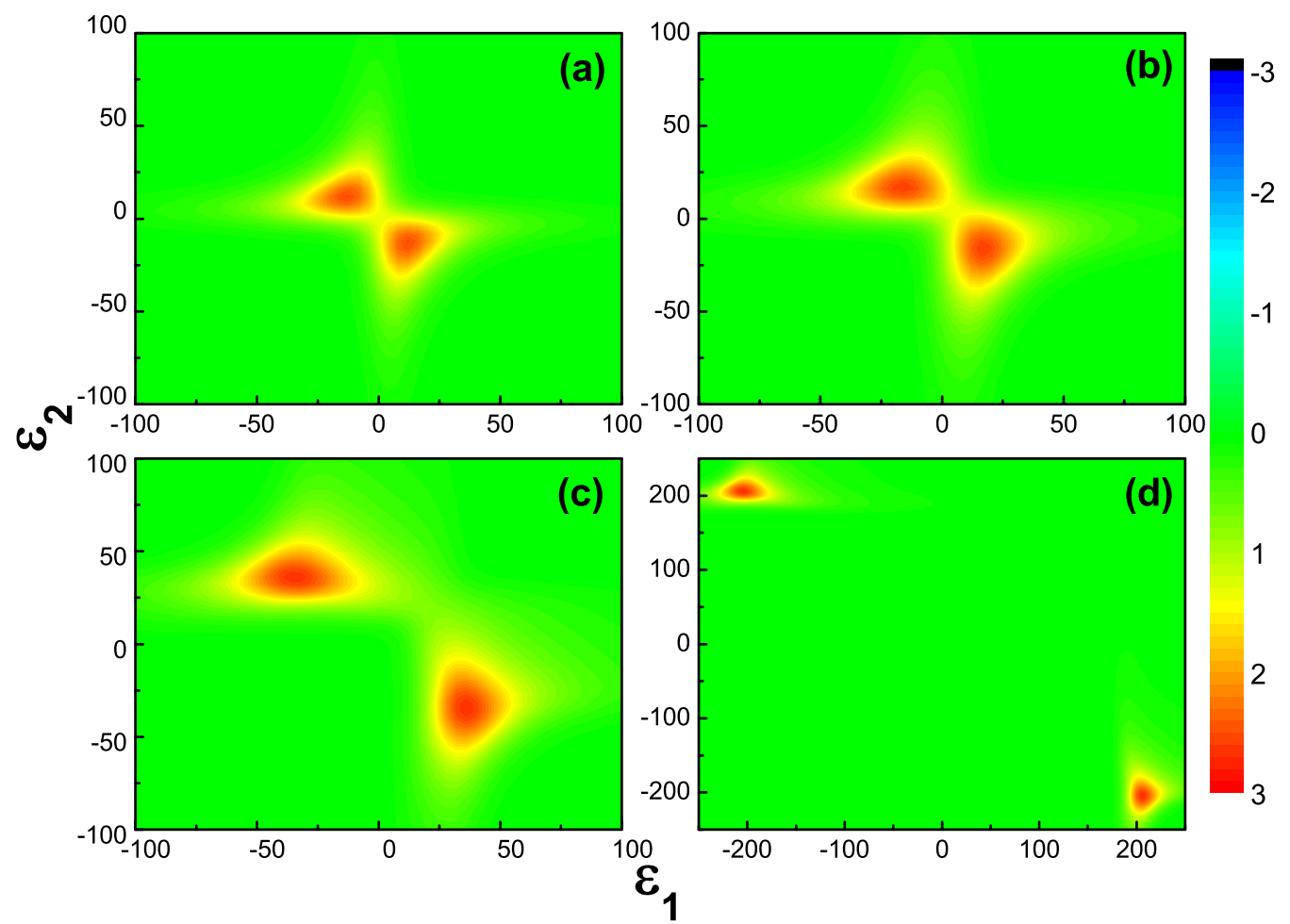

FIG. 5. (Color online) The same as Fig. 4, except that the MBSs are replaced by a normal $s$-wave superconductor. 

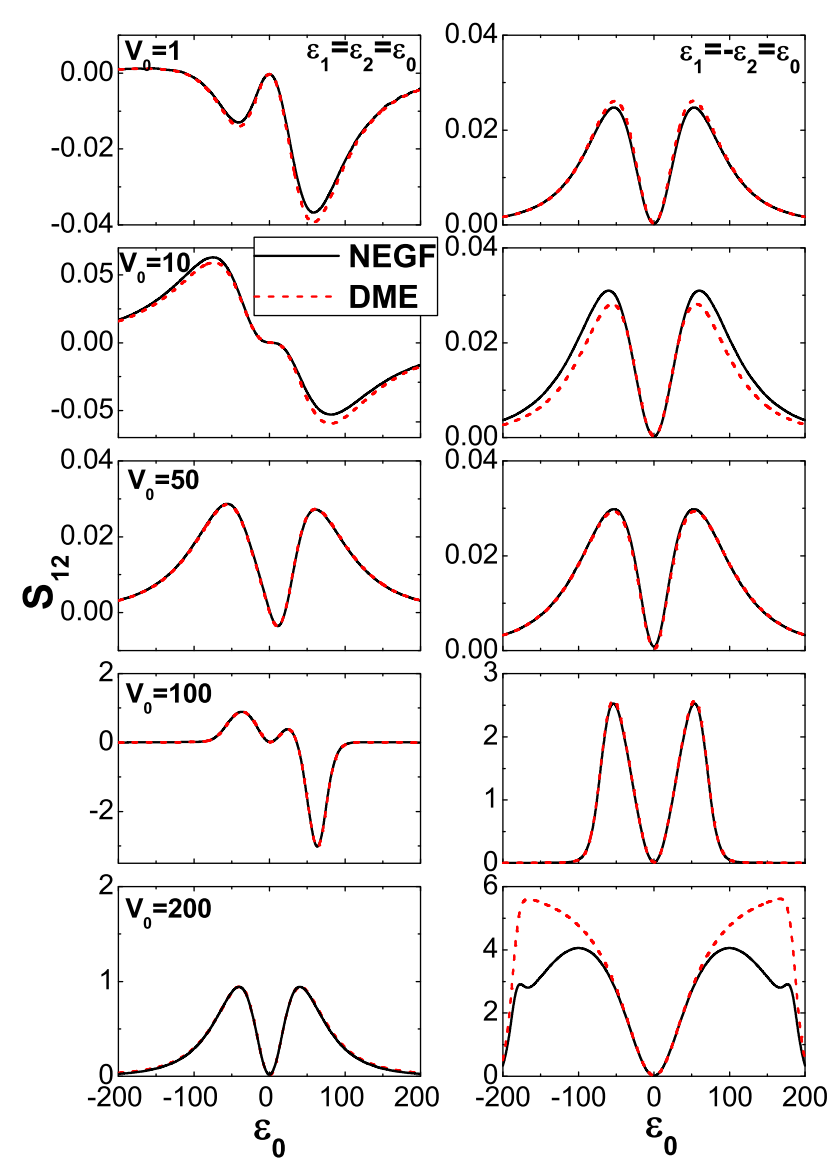

FIG. 6. (Color online) The zero-frequency cross correlation $S_{12}$ as functions of (left) symmetric dot energy levels $\epsilon_{0}\left(=\epsilon_{1}=\epsilon_{2}\right)$ and (right) antisymmetric dot energy levels $\epsilon_{0}\left(=\epsilon_{1}=-\epsilon_{2}\right)$ for different voltages $V_{0}=1,10,50,100$, and 200, respectively. Other parameters are $k_{B} T=5, \lambda_{0}=40$, and $\epsilon_{M}=10$.

In previous studies, the applicability of the DME approach in Majorana-dot hybrid systems is questioned, especially as a function of dot energy level [31]. Above we have demonstrated in a wide range of system parameters that the DME approach could give the numerical results with high accuracy. In principle, there is no extra limitation on the configuration of the dot energy levels when the DME approach is applied. To confirm this point, we present the cross correlation $S_{12}$ calculated by the NEGF method and the DME approach in Fig. 6. We separately consider the symmetric $\left(\epsilon_{1}=\epsilon_{2}\right)$ and antisymmetric $\left(\epsilon_{1}=-\epsilon_{2}\right)$ cases of dot level configurations. In the symmetric configuration $\left(\epsilon_{1}=\epsilon_{2}\right), S_{12}$ shows a strong dependence on the voltage in the leads. For relatively small voltage $V_{0}, S_{12}$ exhibits a pattern of negative values. With the increase of $V_{0}$, the sign reversal of $S_{12}$ appears as a function of $\epsilon_{0}$. It should be noted $S_{12}$ experiences twice sign reversals in the region $\epsilon_{0}>0$, due to the competition between tunneling paths flowing through different eigenlevels. For large $V_{0}$, $S_{12}$ becomes positive eventually. In the antisymmetric level configuration $\left(\epsilon_{1}=-\epsilon_{2}\right), S_{12}$ vanishes at $\epsilon_{i}=0$ and shows two peaks lying on both sides of $\epsilon_{i}=0$. In this case, $S_{12}$ is always positive for different configurations of dot energy levels. Due to the particle-hole symmetry, $S_{12}$ should be symmetric as a function of $\epsilon_{0}$ when changing the sign of $\epsilon_{0}$.

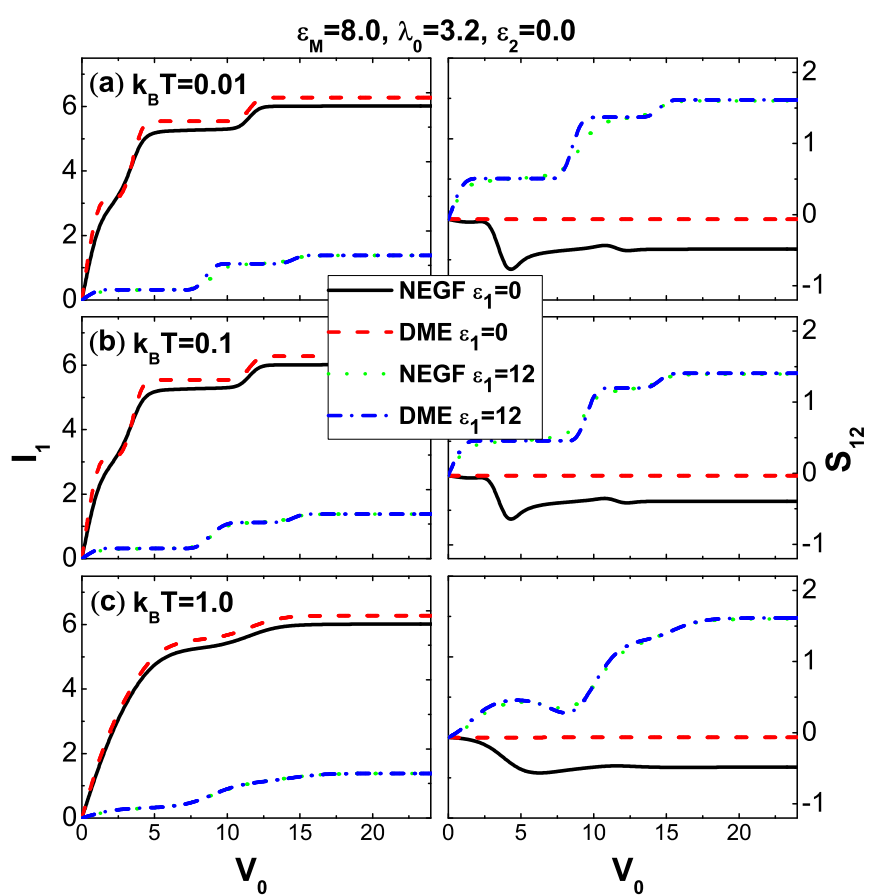

FIG. 7. (Color online) The current $I_{1}$ (left) and zero-frequency cross correlation $S_{12}$ (right) as functions of lead voltage $V_{0}$ for different values of temperature $k_{B} T=0.01,0.1$, and 1 . Two level configurations are examined. One is $\left(\epsilon_{1}, \epsilon_{2}\right)=(0,0)$ and the other is $\left(\epsilon_{1}, \epsilon_{2}\right)=(12,0)$. Other parameters: $\lambda_{0}=3.2$ and $\epsilon_{M}=8$, which are the same as the parameters used in Fig. 2(b) of Ref. [31].

This symmetry can be captured by both the NEGF method and the DME approach, as shown in Fig. 6.

\section{Effect of temperature}

Figures 7 and 8 show the current $I_{1}$ and cross correlation $S_{12}$ as functions of the voltage $V_{0}$ for different temperatures $k_{B} T$. For comparison, we use the parameters from the previous work (Fig. 2 of Ref. [31]). The effect of thermal fluctuation is reflected in the Fermi distributions in both leads. At a low temperature, e.g., $k_{B} T=0.01$, the I-V curves exhibit the staircases and the current jumps to another step when the voltage $V_{0}$ crosses one of the eigenenergies of the dotMBS Hamiltonian. The DME approach assumes the dot-lead coupling as perturbation. In the sequential tunneling regime, the broadening effect in the conductance by the dot-lead coupling is not included in the DME approach. Compared to the results by the NEGF method, the I-V curves by the DME approach display steeper steps at low temperatures. With the increase of temperature, more electrons away from the Fermi energy contribute to the current and thermal fluctuation-induced tunneling is enhanced. For $k_{B} T \gg \Gamma_{0}$, the temperature effect becomes dominant. Therefore, one of the applicable requirements of the DME approach is that the temperature is higher than the dot-lead coupling strength.

At the low temperature $k_{B} T=0.01$, the results by the NEGF method agree well with those by the scattering matrix theory [31]. It is noted that for the case $\epsilon_{1}=\epsilon_{2}=0$, although the currents calculated by the NEGF method and the DME approach agree well with each other, $S_{12}$ shows a large 


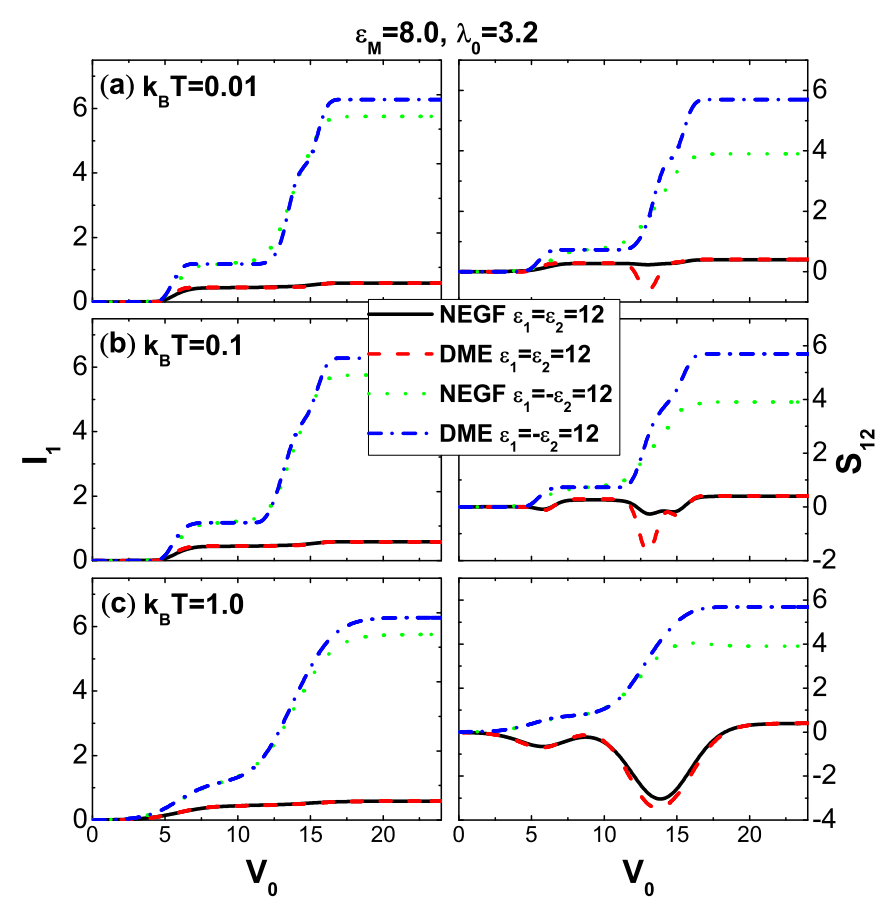

FIG. 8. (Color online) The current $I_{1}$ (left) and zero-frequency cross correlation $S_{12}$ (right) as functions of lead voltage $V_{0}$ for different values of temperature $k_{B} T=0.01,0.1$, and 1 . The symmetric level configuration $\epsilon_{1}=\epsilon_{2}$ and antisymmetric case $\epsilon_{1}=-\epsilon_{2}$ are considered. Other parameters: $\lambda_{0}=3.2$ and $\epsilon_{M}=8$, which is same to the parameters used in Fig. 2 (d) of Ref. [31].

deviation. For different $k_{B} T, S_{12}$ by the DME approach always approaches zero, while the NEGF method gives the negative noise cross correlation. However, for other three dot level configurations, $S_{12}$ by the NEGF method and the DME approach are in good agreement, even at low temperatures. This implies that the DME approach fails in the calculation of current-current correlation when all the eigenenergies and their interval are comparable to the dot-lead coupling strength.

\section{E. Asymmetric dot-Majorana and dot-lead coupling}

In the above discussion we assume a symmetric coupling scenario, i.e., $\Gamma_{1}=\Gamma_{2}$ and $\lambda_{1}=\lambda_{2}$. Actually, the dotMajorana and dot-lead couplings determine the dwell time of an electron through the dots, so asymmetric coupling could also modulate the currents and their correlations. To characterize the asymmetry, we define the parameters

$$
p_{\lambda}=\frac{\lambda_{1}-\lambda_{2}}{\lambda_{1}+\lambda_{2}}
$$

for the dot-Majorana coupling, and

$$
p_{\Gamma}=\frac{\Gamma_{1}-\Gamma_{2}}{\Gamma_{1}+\Gamma_{2}}
$$

for the dot-lead coupling. Figure 4 presents the cross correlation $S_{12}$ as functions of $p_{\lambda}$ and $p_{\Gamma}$. As shown in Fig. 9, the results by two methods show a slight discrepancy for weak dot-Majorana coupling strength $\lambda_{t}=\lambda_{1}+\lambda_{2}$. The dotMajorana interaction $\lambda_{i}$ is the energy scale that measures the relaxation rate of the central region. In the presence of strong

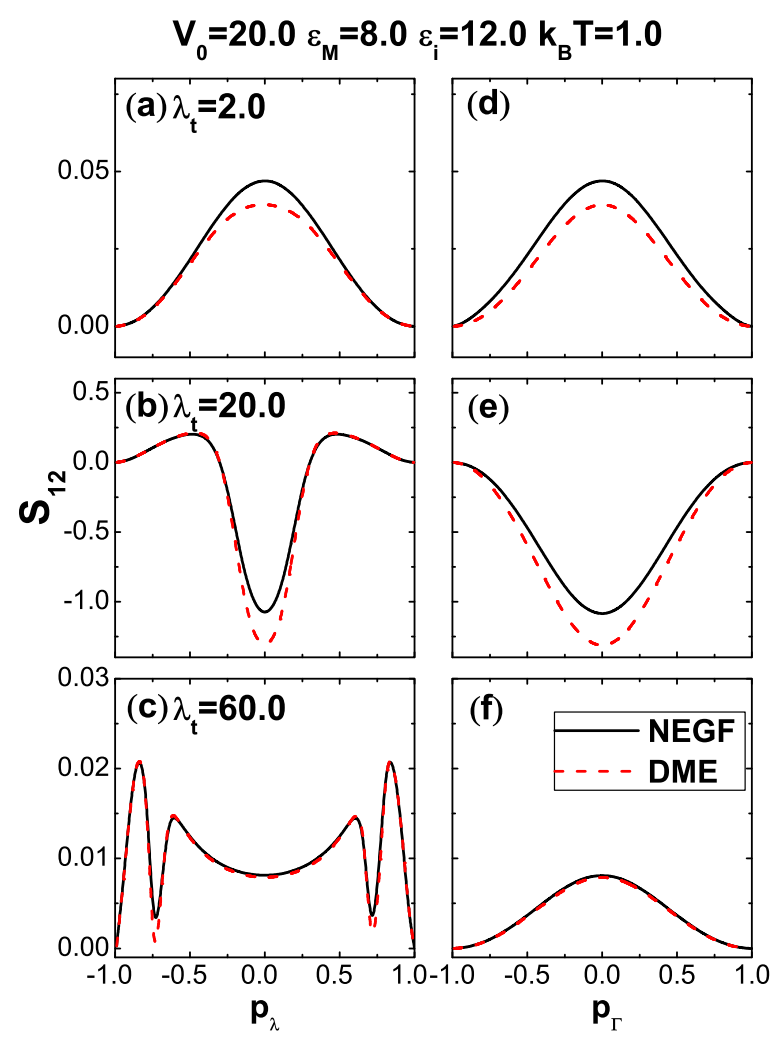

FIG. 9. (Color online) The cross correlation $S_{12}$ as functions of [(a)-(c)] dot-Majorana coupling asymmetry $p_{\lambda}$ and [(d)-(f)] dot-lead coupling asymmetry $p_{\Gamma}$ for different $\lambda_{t}=\lambda_{1}+\lambda_{2}=2.0,20.0$, and 60.0 , respectively. Other parameters are $k_{B} T=1.0, \epsilon_{M}=8.0, \epsilon_{i}=$ 12.0, $V_{0}=20.0$, and $\Gamma_{1}+\Gamma_{2}=2.0$.

asymmetry ( $p_{\lambda} \rightarrow \pm 1$ and $p_{\Gamma} \rightarrow \pm 1$ ), the cross correlation $S_{12}$ is suppressed considerably. For strong dot-Majorana or lead-dot coupling asymmetry, an electron needs more time to tunnel into or out of one of the double dots, resulting in the suppression of the current flowing through the dot and cross correlation. Figure 9 (b) shows that for strong dot-Majorana interaction, the coupling asymmetry $p_{\lambda}$ not only modulates the strength of $S_{12}$, but also could reverse the sign of $S_{12}$.

\section{APPLICABILITY OF DIAGONALIZED MASTER EQUATIONS}

The NEGF method and DME approach are two common tools widely used in mesoscopic tunneling systems [45-50,60-64]. Although the applicability of the NEGF method and the DME approach have been addressed elsewhere $[46,49,65,66]$, it is still unclear for unconventional fermionic systems, such as Majorana fermion system. For this MBS-dot hybrid device, it is of great interest whether the DME method could correctly reflect the information of current and noise, as questioned in a previous paper [31].

In the previous sections we demonstrated in a wide range of system parameters that the DME approach could give the numerical results with high accuracy. However, one still needs to be careful in two cases. The first is that the DME approach assumes that the dot-lead coupling is much weaker than other system energy scales [49]. We demonstrate the importance of 


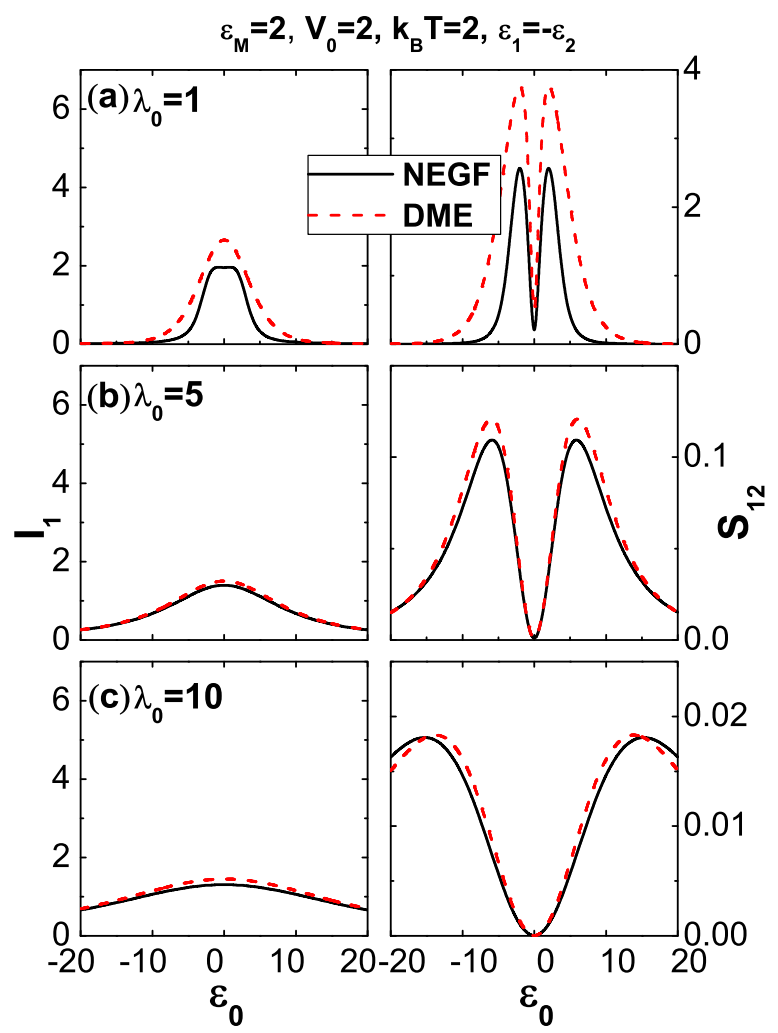

FIG. 10. (Color online) The current $I_{1}$ (left) and zero-frequency cross correlation $S_{12}$ (right) as functions of antisymmetric dot energy level $\epsilon_{0}\left(=\epsilon_{1}=-\epsilon_{2}\right)$ for different voltages $\lambda_{0}=1,5$, and 10 , respectively. Other parameters: $k_{B} T=2, \epsilon_{M}=2$, and $V_{0}=2$.

this restriction in Fig. 10, in which the coherence strength $\lambda_{0}$ takes values of $\Gamma_{0}, 5 \Gamma_{0}$, and $10 \Gamma_{0}$ and $\epsilon_{M}\left(=2 \Gamma_{0}\right)$ is comparable to $\Gamma_{0}$. It is shown that when all the eigenenergies of $H_{0}$ and their intervals are comparable to the dot-lead coupling strength, the results of the DME approach show a clear deviation from those of the NEGF method. The other case is when degenerate eigenstates appear, the matrix for the stationary state population is linearly relevant and DME is not appropriate. This leads to the obvious deviation between the results of the NEGF method and the DME approach which appears at relatively large voltages for the antisymmetric level configuration $\epsilon_{1}=-\epsilon_{2}$ in Figs. 6 and 7. For $\epsilon_{1}=-\epsilon_{2}$, there exists energy degeneracies in the eigenstates, as a result of particle-hole symmetry.

\section{SUMMARY}

In summary, we investigated the currents and their cross correlation in a double dot-Majorana hybrid system by using the NEFG method and the DME approach. We systematically studies the effects of the dot energy levels, Majorana energy splitting, Majorana-dot interaction, and the chemical potential in the leads on the transport properties. In the weak dot-lead coupling regime, the analytical expressions of currents and noise cross correlation are presented. It is found that for the symmetric dot level configuration $\epsilon_{1}=\epsilon_{2}$, the noise cross correlation is negative in the small lead voltage case. With the increase of lead voltage, the cross correlation in the cases of $\epsilon_{i}<0$ and $\epsilon_{i}>0$ turns to positive successively. In contrast, the cross correlation is always positive and symmetric about zero dot level in the antisymmetric case $\epsilon_{1}=-\epsilon_{2}$, as a result of particle-hole symmetry. In addition, the sign of the noise cross correlation is presented as a function of dot energy levels. In the present system, the NEGF method can give the exact solution of the transport properties, which provides a benchmark to check the applicability of the DME approach. The results obtained by both methods agree reasonably well in most cases. However, the results of the DME approach show a clear deviation in the cross correlation for strong dot-lead coupling strength or when energy degeneracy appears in the central region. The comparison will be a reference when generalizing DME to larger systems with many more dot-Majorana units.

\section{ACKNOWLEDGMENT}

This work was supported by the Research Grants Council, University Grants Committee, Hong Kong under Grant No. HKU 703713P and National Natural Science Foundation of China under Grants No. 61474018.

\section{APPENDIX A: FOR NONEQUILIBRIUM GREEN'S FUNCTION METHOD}

In the main context, we present a compact form of NEGF to deduce the formula of tunneling currents and their correlations, which is equivalent to the result of the scattering matrix approach in the noninteracting case. In this Appendix, we will give another set of standard formula of NEGF that has been widely used in previous studies $[42,43,60,67]$. This version of NEGF could take the many-body interaction into account in the proceeding of the equation of motion truncation. In the noninteracting case, it is equivalent to NEGF in the main context. First we introduce the lesser Green's functions, which are defined as

$$
\begin{aligned}
& \left\langle\left\langle c_{\alpha k}(t) \mid d_{\beta}^{\dagger}\left(t^{\prime}\right)\right\rangle\right\rangle^{<}=i\left\langle d_{\beta}^{\dagger}\left(t^{\prime}\right) c_{\alpha k}(t)\right\rangle, \\
& \left\langle\left\langle d_{\alpha}(t) \mid c_{\beta k}^{\dagger}\left(t^{\prime}\right)\right\rangle\right\rangle^{<}=i\left\langle c_{\beta k}^{\dagger}\left(t^{\prime}\right) d_{\alpha}(t)\right\rangle .
\end{aligned}
$$

In terms of the lesser Green's functions, the current is written into

$$
I_{\alpha}(t)=\frac{e}{\hbar} t_{\alpha} \sum_{k}\left[\left\langle\left\langle d_{\alpha}(t) \mid c_{\alpha k}^{\dagger}(t)\right\rangle\right\rangle^{<}-\left\langle\left\langle c_{\alpha k}(t) \mid d_{\alpha}^{\dagger}(t)\right\rangle\right\rangle^{<}\right],
$$

and the cross correlation $S_{12}$ could be expressed as

$$
\begin{aligned}
S_{12}\left(t-t^{\prime}\right)= & \frac{2 e^{2}}{\hbar^{2}} t_{1} t_{2} \sum_{k, k^{\prime}}\left[\left\langle\left\langle d_{2}\left(t^{\prime}\right) \mid c_{1 k}^{\dagger}(t)\right\rangle\right\rangle^{<}\left\langle\left\langle c_{2 k^{\prime}}^{\dagger}\left(t^{\prime}\right) \mid d_{1}(t)\right\rangle\right\rangle^{<}\right. \\
& +\left\langle\left\langle c_{2 k^{\prime}}\left(t^{\prime}\right) \mid d_{1}^{\dagger}(t)\right\rangle\right\rangle^{<}\left\langle\left\langle d_{2}^{\dagger}\left(t^{\prime}\right) \mid c_{1 k}(t)\right\rangle\right\rangle^{<} \\
& +\left\langle\left\langle c_{2 k^{\prime}}^{\dagger}\left(t^{\prime}\right) \mid d_{1}^{\dagger}(t)\right\rangle\right\rangle^{<}\left\langle\left\langle d_{2}\left(t^{\prime}\right) \mid c_{1 k}(t)\right\rangle\right\rangle^{<} \\
& +\left\langle\left\langle d_{2}^{\dagger}\left(t^{\prime}\right) \mid c_{1 k}^{\dagger}(t)\right\rangle\right\rangle^{<}\left\langle\left\langle c_{2 k^{\prime}}\left(t^{\prime}\right) \mid d_{1}(t)\right\rangle\right\rangle^{<} \\
& -\left\langle\left\langle c_{2 k^{\prime}}^{\dagger}\left(t^{\prime}\right) \mid c_{1 k}^{\dagger}(t)\right\rangle\right\rangle^{<}\left\langle\left\langle d_{2}\left(t^{\prime}\right) \mid d_{1}(t)\right\rangle\right\rangle^{<} \\
& -\left\langle\left\langle c_{2 k^{\prime}}\left(t^{\prime}\right) \mid c_{1 k}(t)\right\rangle\right\rangle^{<}\left\langle\left\langle d_{2}^{\dagger}\left(t^{\prime}\right) \mid d_{1}^{\dagger}(t)\right\rangle\right\rangle^{<} \\
& -\left\langle\left\langle c_{2 k^{\prime}}^{\dagger}\left(t^{\prime}\right) \mid c_{1 k}(t)\right\rangle\right\rangle^{<}\left\langle\left\langle d_{2}\left(t^{\prime}\right) \mid d_{1}^{\dagger}(t)\right\rangle\right\rangle^{<} \\
& \left.-\left\langle\left\langle c_{2 k^{\prime}}\left(t^{\prime}\right) \mid c_{1 k}^{\dagger}(t)\right\rangle\right\rangle^{<}\left\langle\left\langle d_{2}^{\dagger}\left(t^{\prime}\right) \mid d_{1}(t)\right\rangle\right\rangle^{<}\right] . \quad \text { (A3) }
\end{aligned}
$$


In the above equations, we have approximately truncated the four-operator correlation functions [67], e.g.,

$$
\begin{aligned}
& \left\langle c_{\alpha k}^{\dagger} d_{\alpha} c_{\beta k^{\prime}}^{\dagger} d_{\beta}\right\rangle \approx\left\langle c_{\alpha k}^{\dagger} d_{\beta}\right\rangle\left\langle d_{\alpha} c_{\beta k^{\prime}}^{\dagger}\right\rangle-\left\langle c_{\alpha k}^{\dagger} c_{\beta k^{\prime}}^{\dagger}\right\rangle\left\langle d_{\alpha} d_{\beta}\right\rangle+\left\langle c_{\alpha k}^{\dagger} d_{\alpha}\right\rangle\left\langle c_{\beta k^{\prime}}^{\dagger} d_{\beta}\right\rangle, \\
& \left\langle c_{\alpha k}^{\dagger} d_{\alpha} d_{\beta}^{\dagger} c_{\beta k^{\prime}}\right\rangle \approx\left\langle c_{\alpha k}^{\dagger} c_{\beta k^{\prime}}\right\rangle\left\langle d_{\alpha} d_{\beta}^{\dagger}\right\rangle-\left\langle c_{\alpha k}^{\dagger} d_{\beta}^{\dagger}\right\rangle\left\langle d_{\alpha} c_{\beta k^{\prime}}\right\rangle+\left\langle c_{\alpha k}^{\dagger} d_{\alpha}\right\rangle\left\langle d_{\beta}^{\dagger} c_{\beta k^{\prime}}\right\rangle .
\end{aligned}
$$

After applying a Fourier transform, the stationary current can be expressed as

$$
I_{\alpha}=\frac{e}{\hbar} \int d \omega\left[t_{\alpha}\left\langle\left\langle d_{\alpha} \mid c_{\alpha k}^{\dagger}\right\rangle\right\rangle^{<}-t_{\alpha}\left\langle\left\langle c_{\alpha k} \mid d_{\alpha}^{\dagger}\right\rangle\right\rangle^{<}\right],
$$

and the cross correlation spectrum is given by

$$
\begin{aligned}
S_{12}(\omega)= & \int d t S_{12}(t) e^{i \omega t} \\
= & \frac{4 \pi e^{2}}{\hbar^{2}} t_{1} t_{2} \int d \omega^{\prime} \sum_{k, k^{\prime}}\left[\left\langle\left\langle d_{2} \mid c_{1 k}^{\dagger}\right\rangle\right\rangle_{\omega^{\prime}+\omega}^{<}\left\langle\left\langle c_{2 k^{\prime}}^{\dagger} \mid d_{1}\right\rangle\right\rangle_{\omega^{\prime}}^{<}+\left\langle\left\langle c_{2 k^{\prime}} \mid d_{1}^{\dagger}\right\rangle\right\rangle_{\omega^{\prime}+\omega^{\prime}}^{<}\left\langle\left\langle d_{2}^{\dagger} \mid c_{1 k}\right\rangle\right\rangle_{\omega^{\prime}}^{<}\right. \\
& +\left\langle\left\langle c_{2 k^{\prime}}^{\dagger} \mid d_{1}^{\dagger}\right\rangle\right\rangle_{\omega^{\prime}+\omega}^{<}\left\langle\left\langle d_{2} \mid c_{1 k}\right\rangle\right\rangle_{\omega^{\prime}}^{<}+\left\langle\left\langle d_{2}^{\dagger} \mid c_{1 k}^{\dagger}\right\rangle\right\rangle_{\omega^{\prime}+\omega^{\prime}}^{<}\left\langle\left\langle c_{2 k^{\prime}} \mid d_{1}\right\rangle\right\rangle_{\omega^{\prime}}^{<}-\left\langle\left\langle c_{2 k^{\prime}}^{\dagger} \mid c_{1 k}^{\dagger}\right\rangle\right\rangle_{\omega^{\prime}+\omega}^{<}\left\langle\left\langle d_{2} \mid d_{1}\right\rangle\right\rangle_{\omega^{\prime}}^{<} \\
& \left.-\left\langle\left\langle c_{2 k^{\prime}} \mid c_{1 k}\right\rangle\right\rangle_{\omega^{\prime}}^{<}\left\langle\left\langle d_{2}^{\dagger} \mid d_{1}^{\dagger}\right\rangle\right\rangle_{\omega^{\prime}+\omega}^{<}-\left\langle\left\langle c_{2 k^{\prime}}^{\dagger} \mid c_{1 k}\right\rangle\right\rangle_{\omega^{\prime}}^{<}\left\langle\left\langle d_{2} \mid d_{1}^{\dagger}\right\rangle\right\rangle_{\omega^{\prime}+\omega}^{<}-\left\langle\left\langle c_{2 k^{\prime}} \mid c_{1 k}^{\dagger}\right\rangle\right\rangle_{\omega^{\prime}+\omega^{\prime}}^{<}\left\langle\left\langle d_{2}^{\dagger} \mid d_{1}\right\rangle\right\rangle_{\omega^{\prime}}^{<}\right],
\end{aligned}
$$

where the Green's functions involving both dot and lead operators can be readily related to the Green's functions of only dot operators and only lead operators. By applying the Langreth analytic continuation rules [42,43]

$$
\begin{aligned}
& \left\langle\left\langle d_{\alpha}^{(\dagger)} \mid c_{\beta k}^{\dagger}\right\rangle\right\rangle^{<}=t_{\beta}\left[\left\langle\left\langle d_{\alpha}^{(\dagger)} \mid d_{\beta}^{\dagger}\right\rangle\right\rangle^{r}\left\langle\left\langle c_{\beta k} \mid c_{\beta k}^{\dagger}\right\rangle\right\rangle^{0<}+\left\langle\left\langle d_{\alpha}^{(\dagger)} \mid d_{\beta}^{\dagger}\right\rangle\right\rangle^{<}\left\langle\left\langle c_{\beta k} \mid c_{\beta k}^{\dagger}\right\rangle\right\rangle^{0 a}\right], \\
& \left\langle\left\langle d_{\alpha}^{(\dagger)} \mid c_{\beta k}\right\rangle\right\rangle^{<}=-t_{\beta}\left[\left\langle\left\langle d_{\alpha}^{(\dagger)} \mid d_{\beta}\right\rangle\right\rangle^{r}\left\langle\left\langle c_{\beta k}^{\dagger} \mid c_{\beta k}\right\rangle\right\rangle^{0<}+\left\langle\left\langle d_{\alpha}^{(\dagger)} \mid d_{\beta}\right\rangle\right\rangle^{<}\left\langle\left\langle c_{\beta k}^{\dagger} \mid c_{\beta k}\right\rangle\right\rangle^{0 a}\right], \\
& \left\langle\left\langle c_{\beta k}^{\dagger} \mid d_{\alpha}^{(\dagger)}\right\rangle\right\rangle^{<}=t_{\beta}\left[\left\langle\left\langle d_{\beta}^{\dagger} \mid d_{\alpha}^{(\dagger)}\right\rangle\right\rangle^{r}\left\langle\left\langle c_{\beta k}^{\dagger} \mid c_{\beta k}\right\rangle\right\rangle^{0<}+\left\langle\left\langle d_{\beta}^{\dagger} \mid d_{\alpha}^{(\dagger)}\right\rangle\right\rangle^{<}\left\langle\left\langle c_{\beta k}^{\dagger} \mid c_{\beta k}\right\rangle\right\rangle^{0 a}\right], \\
& \left\langle\left\langle c_{\beta k} \mid d_{\alpha}^{(\dagger)}\right\rangle\right\rangle^{<}=-t_{\beta}\left[\left\langle\left\langle d_{\beta} \mid d_{\alpha}^{(\dagger)}\right\rangle\right\rangle^{r}\left\langle\left\langle c_{\beta k} \mid c_{\beta k}^{\dagger}\right\rangle\right\rangle^{0<}+\left\langle\left\langle d_{\beta} \mid d_{\alpha}^{(\dagger)}\right\rangle\right\rangle^{<}\left\langle\left\langle c_{\beta k} \mid c_{\beta k}^{\dagger}\right\rangle\right\rangle^{0 a}\right],
\end{aligned}
$$

where $\left\langle\left\langle c_{\alpha k} \mid c_{\beta k}^{\dagger}\right\rangle\right\rangle^{0}$ is the bare Green's functions for the leads. For the present model, the exotic contact terms $\left\langle\left\langle c_{\alpha k} \mid c_{\beta k^{\prime}}\right\rangle\right\rangle^{<}$and $\left\langle\left\langle c_{\alpha k}^{\dagger} \mid c_{\beta k^{\prime}}^{\dagger}\right\rangle\right\rangle^{<}$are also kept due to the pairing effect induced by MBSs and they could be obtained from dot-lead Green's functions

$$
\begin{aligned}
& \left\langle\left\langle c_{\alpha k}^{(\dagger)} \mid c_{\beta k^{\prime}}^{\dagger}\right\rangle\right\rangle^{<}=t_{\beta}\left[\left\langle\left\langle c_{\alpha k}^{(\dagger)} \mid d_{\beta}^{\dagger}\right\rangle\right\rangle^{r}\left\langle\left\langle c_{\beta k^{\prime}} \mid c_{\beta k^{\prime}}^{\dagger}\right\rangle\right\rangle^{0<}+\left\langle\left\langle c_{\alpha k}^{(\dagger)} \mid d_{\beta}^{\dagger}\right\rangle\right\rangle^{<}\left\langle\left\langle c_{\beta k^{\prime}} \mid c_{\beta k^{\prime}}^{\dagger}\right\rangle\right\rangle^{0 a}\right], \\
& \left\langle\left\langle c_{\alpha k}^{(\dagger)} \mid c_{\beta k^{\prime}}\right\rangle\right\rangle^{<}=-t_{\beta}\left[\left\langle\left\langle c_{\alpha k}^{(\dagger)} \mid d_{\beta}\right\rangle\right\rangle^{r}\left\langle\left\langle c_{\beta k^{\prime}}^{\dagger} \mid c_{\beta k^{\prime}}\right\rangle\right\rangle^{0<}+\left\langle\left\langle c_{\alpha k}^{(\dagger)} \mid d_{\beta}\right\rangle\right\rangle^{<}\left\langle\left\langle c_{\beta k^{\prime}}^{\dagger} \mid c_{\beta k^{\prime}}\right\rangle\right\rangle^{0 a}\right] .
\end{aligned}
$$

The bare Green's functions for the leads are given by

$$
\begin{aligned}
\left\langle\left\langle c_{\alpha k} \mid c_{\alpha k}^{\dagger}\right\rangle\right\rangle_{\omega}^{0<} & =i \pi f(\omega) \delta\left(\omega-\epsilon_{\alpha k}\right), \\
\left\langle\left\langle c_{\alpha k}^{\dagger} \mid c_{\alpha k}\right\rangle\right\rangle_{\omega}^{0<} & =i \pi[1-f(-\omega)] \delta\left(\omega+\epsilon_{\alpha k}\right), \\
\left\langle\left\langle c_{\alpha k} \mid c_{\alpha k}^{\dagger}\right\rangle\right\rangle_{\omega}^{0 r(a)} & =\mp i \pi \delta\left(\omega-\epsilon_{\alpha k}\right), \\
\left\langle\left\langle c_{\alpha k}^{\dagger} \mid c_{\alpha k}\right\rangle\right\rangle_{\omega}^{0 r(a)} & =\mp i \pi \delta\left(\omega+\epsilon_{\alpha k}\right) .
\end{aligned}
$$

Performing the standard equation of motion procedure for the central region, we could obtain the retarded Green's functions in the Nambu space spanned by $\psi=\left(d_{1}, d_{2}, f, d_{1}^{\dagger}, d_{2}^{\dagger}, f^{\dagger}\right)^{T}$,

$$
\mathbf{M G}^{r}=1
$$

where

$$
\mathbf{M}=\left(\begin{array}{cccccc}
\omega-\epsilon_{1}+i \Gamma_{1} & 0 & -\lambda_{1} & 0 & 0 & -\lambda_{1} \\
0 & \omega-\epsilon_{2}+i \Gamma_{2} & -\lambda_{2} & 0 & 0 & \lambda_{2} \\
-\lambda_{1} & -\lambda_{2} & \omega-\epsilon_{M} & \lambda_{1} & -\lambda_{2} & 0 \\
0 & 0 & \lambda_{1} & \omega+\epsilon_{1}+i \Gamma_{1} & 0 & \lambda_{1} \\
0 & 0 & -\lambda_{2} & 0 & \omega+\epsilon_{2}+i \Gamma_{2} & \lambda_{2} \\
-\lambda_{1} & \lambda_{2} & 0 & \lambda_{1} & \lambda_{2} & \omega+\epsilon_{M}
\end{array}\right)
$$

and $G^{r}=\left\langle\left\langle\psi \mid \psi^{\dagger}\right\rangle\right\rangle^{r}$. 
Using the relationship $\mathbf{G}^{r}=\left(\mathbf{G}^{a}\right)^{\dagger}$, we can obtain the advanced Green's function. The related lesser Green's functions can be calculated by the Keldysh equation $G^{<}=G^{r} \Sigma^{<} G^{a}$. In the present representation, the self energy $\Sigma^{<}$is

$$
\Sigma^{<}(\omega)=\left(\begin{array}{cc}
\Sigma_{e}^{<} & 0 \\
0 & \Sigma_{h}^{<}
\end{array}\right),
$$

with

$$
\Sigma_{e(h)}^{<}=\left(\begin{array}{ccc}
i \Gamma_{1} f\left(\omega \mp \mu_{1}\right) & 0 & 0 \\
0 & i \Gamma_{2} f\left(\omega \mp \mu_{2}\right) & 0 \\
0 & 0 & 0
\end{array}\right),
$$

where $f(\omega)=\left[1+e^{\omega / k_{B} T}\right]^{-1}$ is the Fermi distribution function and $k_{B} T$ is the system temperature.

\section{APPENDIX B: FOR DIAGONALIZED MASTER EQUATION APPROACH}

For the central region, we can diagonalize the Hamiltonian $H_{0}=H_{D}+H_{M}+H_{D M}$ by solving their eigenenergies and the corresponding eigenstates. For every state, we have

$$
\begin{aligned}
& H_{0}|000\rangle=-\frac{\epsilon_{M}}{2}|000\rangle+\lambda_{1}|101\rangle-\lambda_{2}|011\rangle, \\
& H_{0}|101\rangle=\left(\epsilon_{1}+\frac{\epsilon_{M}}{2}\right)|101\rangle+\lambda_{1}|000\rangle+\lambda_{2}|110\rangle, \\
& H_{0}|011\rangle=\left(\epsilon_{2}+\frac{\epsilon_{M}}{2}\right)|011\rangle-\lambda_{1}|110\rangle-\lambda_{2}|000\rangle, \\
& H_{0}|110\rangle=\left(\epsilon_{1}+\epsilon_{2}-\frac{\epsilon_{M}}{2}\right)|110\rangle-\lambda_{1}|011\rangle+\lambda_{2}|101\rangle .
\end{aligned}
$$

It can be seen that the above four states $|000\rangle,|101\rangle,|011\rangle$, and $|110\rangle$ form a closed block of even parity. Similarly, another four states $|001\rangle,|100\rangle,|010\rangle$, and $|111\rangle$ form a closed block of odd parity and they meet

$$
\begin{aligned}
& H_{0}|001\rangle=\frac{\epsilon_{M}}{2}|001\rangle+\lambda_{1}|100\rangle+\lambda_{2}|010\rangle, \\
& H_{0}|100\rangle=\left(\epsilon_{1}-\frac{\epsilon_{M}}{2}\right)|101\rangle+\lambda_{1}|001\rangle-\lambda_{2}|111\rangle,
\end{aligned}
$$

$$
\begin{aligned}
& H_{0}|010\rangle=\left(\epsilon_{2}-\frac{\epsilon_{M}}{2}\right)|011\rangle-\lambda_{1}|111\rangle+\lambda_{2}|001\rangle, \\
& H_{0}|111\rangle=\left(\epsilon_{1}+\epsilon_{2}+\frac{\epsilon_{M}}{2}\right)|111\rangle-\lambda_{1}|010\rangle-\lambda_{2}|100\rangle .
\end{aligned}
$$

It should also be noted that for the tunnel operators, the secondquantization operators in Eq. (23) can be written in the relevant many-body basis as

$$
\begin{aligned}
& d_{1}=|001\rangle\langle 101|+| 010\rangle\langle 110|+| 000\rangle\langle 100|+| 011\rangle\langle 111|, \\
& d_{1}^{\dagger}=|101\rangle\langle 001|+| 110\rangle\langle 010|+| 100\rangle\langle 000|+| 111\rangle\langle 011|, \\
& d_{2}=|001\rangle\langle 011|-| 100\rangle\langle 110|+| 000\rangle\langle 010|-| 101\rangle\langle 111|, \\
& d_{2}^{\dagger}=|011\rangle\langle 001|-| 110\rangle\langle 100|+| 010\rangle\langle 000|-| 111\rangle\langle 101| .
\end{aligned}
$$

Based on these relationships, we could obtain the matrix form of the current operators in Eq. (23) in the diagonalized representation. Correspondingly, the statistical averaging of any time-dependent operator $\hat{A}(t)$ becomes

$$
\langle\hat{A}(t)\rangle=\operatorname{Tr}\{\hat{A} \rho\}=\sum_{k}[\boldsymbol{A} \boldsymbol{\rho}(t)]_{k}=\sum_{k}\left[\boldsymbol{A} \boldsymbol{\rho}^{(0)}\right]_{k},
$$

where $\boldsymbol{A}$ is the matrix expression of the operator $\hat{A}, \rho^{(0)}$ is the steady state solution of the rate equations, and $[\rho]_{k}$ is the $k$ th element of the vector $\rho$.

\section{APPENDIX C: CURRENTS AND CROSS CORRELATION IN WEAK DOT-LEAD COUPLING LIMIT}

For weak dot-lead coupling strength, the expressions of the current and noise cross correlation can be found with the help of NEGF. For simplicity, we consider the symmetric dotleading and dot-Majorana coupling strength, i.e., $\Gamma_{i}=\Gamma_{0}$ and $\lambda_{i}=\lambda_{0}$. A symmetric lead voltages $V_{i}=V_{0}$ is applied in both leads.

In the symmetric level configuration $\epsilon_{1}=\epsilon_{2}$,

$$
\begin{gathered}
I_{i} \approx \int d \omega \frac{8 \lambda_{0}^{4} \Gamma_{0}^{2}\left[f_{\omega}^{-}-f_{\omega}^{+}\right]\left[\left(\epsilon_{M}^{2}+\omega^{2}\right)\left(\epsilon_{0}^{2}-\omega^{2}\right)^{2}+8 \lambda_{0}^{2} \omega^{2}\left(2 \lambda_{0}^{2}+\epsilon_{0}^{2}-\omega^{2}\right)\right]}{A_{\omega}^{2}+B_{\omega}^{2}}, \\
S_{12} \approx \int d \omega \frac{16 \epsilon_{M}^{2} \lambda_{0}^{4} \Gamma_{0}^{2}\left[\left(\epsilon_{0}^{2}-\omega^{2}\right)^{2}\left(f_{\omega}^{-}-f_{\omega}^{+}\right)^{2}+4 \epsilon_{0} \omega\left[\left(\epsilon_{0}+\omega\right)^{2}\left(f_{\omega}^{-}-1\right) f_{\omega}^{-}-\left(\epsilon_{0}-\omega\right)^{2}\left(f_{\omega}^{+}-1\right) f_{\omega}^{+}\right]\right]}{\left[A_{\omega}+B_{\omega}^{2} / A_{\omega}\right]^{2}},
\end{gathered}
$$

where $f_{\omega}^{ \pm}=\left[1+e^{\left(\omega \pm V_{0}\right) / k_{B} T}\right]^{-1}$,

$$
\begin{aligned}
& A_{\omega}=\left(\omega^{2}-\epsilon_{M}^{2}\right)\left(\epsilon_{0}^{2}-\omega^{2}\right)^{2}+8 \lambda_{0}^{2} \omega^{2}\left(2 \lambda_{0}^{2}+\epsilon_{0}^{2}-\omega^{2}\right), \\
& B_{\omega}=2 \Gamma_{0} \omega\left[\left(\omega^{2}-4 \lambda_{0}^{2}-\epsilon_{0}^{2}\right)\left(\omega^{2}-2 \lambda_{0}^{2}-\epsilon_{M}^{2}\right)-4 \lambda_{0}^{2} \epsilon_{M}^{2}\right] .
\end{aligned}
$$

In the limit of large lead voltage, $f_{\omega}^{-}=1, f_{\omega}^{+}=0$, then one obtains Eq. (30). In the limit of small lead voltage, $f_{\omega}^{-} \approx f_{\omega}^{+}=$ $0 \equiv f_{\omega}$, then one obtains Eq. (32). Moreover, by using the relationship $f_{\omega}^{-}=1-f_{-\omega}^{+}$, one can check that $S_{12}\left(\epsilon_{0}\right)<S_{12}\left(-\epsilon_{0}\right)$ for $\epsilon_{0}>0$ for any $V_{0}$. 
In the antisymmetric level configuration $\epsilon_{1}=-\epsilon_{2}$,

$$
\begin{gathered}
I_{i} \approx \int d \omega \frac{8 \lambda_{0}^{4} \Gamma_{0}^{2}\left[f_{\omega}^{-}-f_{\omega}^{+}\right]\left[\left(\epsilon_{M}^{2}+\omega^{2}\right)\left(\epsilon_{0}^{2}-\omega^{2}\right)^{2}+8 \lambda_{0}^{2} \omega^{2}\left(2 \lambda_{0}^{2}+\epsilon_{0}^{2}-\omega^{2}\right)+8 \epsilon_{0}^{2} \epsilon_{M}^{2} \omega^{2}\right]}{A_{\omega}^{2}+B_{\omega}^{2}}, \\
S_{12} \approx \int d \omega \frac{16 \epsilon_{M}^{2} \lambda_{0}^{4} \Gamma_{0}^{2}\left[\left(\epsilon_{0}^{2}-\omega^{2}\right)^{2}\left(f_{\omega}^{-}-f_{\omega}^{+}\right)^{2}+8 \epsilon_{0}^{2} \omega^{2}\left(f_{\omega}^{-}+f_{\omega}^{+}-2 f_{\omega}^{-} f_{\omega}^{+}\right)\right]}{\left[A_{\omega}+B_{\omega}^{2} / A_{\omega}\right]^{2}},
\end{gathered}
$$

from which, we can obtain Eqs. (33) and (34) for large and small lead voltages, respectively.

\section{APPENDIX D: THE MODEL WITH MBS REPLACED BY A NORMAL SUPERCONDUCTOR}

For comparison, we also consider a non-Majorana setup. It consists of a superconductor coupled to two quantum dots, and each dot is connected to a normal metallic electrode. The Hamiltonian of the double dots with on-site Coulomb repulsion is described by

$$
H_{D D}=\sum_{i \sigma} \epsilon_{i \sigma} d_{i \sigma}^{\dagger} d_{i \sigma}+\sum_{i} U_{i} n_{i \uparrow} n_{i \downarrow},
$$

where $i(=1,2)$ represents the dot index and the on-site Coulomb interactions are measured by $U_{i}$. The Hamiltonian of the superconducting lead reads

$$
H_{S C}=\sum_{k \sigma} \epsilon_{s k \sigma} c_{s k \sigma}^{\dagger} c_{s k \sigma}+\sum_{k} \Delta\left(c_{s k \uparrow}^{\dagger} c_{s-k \downarrow}+\text { H.c. }\right)
$$

with the lead-electron operators $c_{s k \sigma}^{\dagger}$ and $c_{s k \sigma}$. The superconductivity in the lead is described by the pairing order parameter $\Delta$. The electrochemical potential of the superconductor is taken as the reference for energies and set to zero, i.e., $\mu_{S}=0$. The Hamiltonian describing the tunneling between the superconducting lead and the double dot is given by

$$
H_{T S}=\sum_{i k \sigma}\left[t_{s i} c_{s k \sigma}^{\dagger} d_{i \sigma}+\text { H.c. }\right] \text {. }
$$

Assuming that the normal-state density of states in the superconducting lead $\rho_{s}$ is constant in the energy window relevant for transport, we define the coupling strength $\Gamma_{s i}=$ $2 \pi \rho_{s}\left|t_{s i}\right|^{2}$ for the tunneling between the superconductor and dot $i$. In the case that $\Delta$ is much stronger than other system parameters, the quasiparticles in the superconductor are inaccessible, and one can trace out of the degrees of freedom of the superconducting lead without inducing any dissipative dynamics. By performing a real-time perturbative expansion and adding up all contributions in $\Gamma_{s i}$, we obtain the following effective Hamiltonian describing the dynamics of the double dots [58,59]

$$
\begin{aligned}
H_{\mathrm{eff}}= & H_{D D}-\sum_{i} \frac{\Gamma_{s i}}{2}\left(d_{i \uparrow}^{\dagger} d_{i \downarrow}^{\dagger}+\text { H.c. }\right) \\
& +\frac{\sqrt{\Gamma_{s 1} \Gamma_{s 2}}}{2}\left(d_{1 \uparrow}^{\dagger} d_{2 \downarrow}^{\dagger}-d_{2 \uparrow}^{\dagger} d_{1 \downarrow}^{\dagger}+\text { H.c. }\right),
\end{aligned}
$$

where two new contributions are added to the double-dot Hamiltonian. The first one describes the local Andreev reflection for each dot. The second term describes the formation of nonlocal superconducting correlations between the two dots induced by the splitting of Cooper pairs into the two dots. In realistic devices, $U_{i}$ is of the order of $1 \mathrm{meV}$ and is much larger than the other energy scales relevant for transport. We calculate the cross correlation for this setup in the limit $U_{i} \rightarrow \infty$ (so that each dot is singly occupied) and the results are shown in Fig. 5.
[1] E. Majorana, Nuovo Cimento 14, 171 (1937).

[2] C. Nayak, S. H. Simon, A. Stern, M. Freedman, and S. Das Sarma, Rev. Mod. Phys. 80, 1083 (2008).

[3] A. Stern, Nature (London) 464, 187 (2010).

[4] D. A. Ivanov, Phys. Rev. Lett. 86, 268 (2001).

[5] A. Kitaev, Ann. Phys. (NY) 303, 2 (2003).

[6] G. Moore and N. Read, Nucl. Phys. B 360, 362 (1991).

[7] K. Flensberg, Phys. Rev. Lett. 106, 090503 (2011).

[8] L. Fu, Phys. Rev. Lett. 104, 056402 (2010).

[9] J. D. Sau, R. M. Lutchyn, S. Tewari, and S. Das Sarma, Phys. Rev. Lett. 104, 040502 (2010).

[10] J. Alicea, Y. Oreg, G. Refael, F. von Oppen, and M. P. A. Fisher, Nat. Phys. 7, 412 (2011).

[11] L. Jiang, C. L. Kane, and J. Preskill, Phys. Rev. Lett. 106, 130504 (2011).

[12] P. Bonderson and R. M. Lutchyn, Phys. Rev. Lett. 106, 130505 (2011).

[13] Y. Oreg, G. Refael, and F. von Oppen, Phys. Rev. Lett. 105, 177002 (2010).
[14] R. M. Lutchyn, J. D. Sau, and S. Das Sarma, Phys. Rev. Lett. 105, 077001 (2010).

[15] J. Alicea, Phys. Rev. B 81, 125318 (2010).

[16] J. D. Sau, S. Tewari, R. M. Lutchyn, T. D. Stanescu, and S. Das Sarma, Phys. Rev. B 82, 214509 (2010).

[17] C. W. J. Beenakker, Annu. Rev. Con. Mat. Phys. 4, 113 (2013).

[18] V. Mourik, K. Zuo, S. M. Frolov, S. R. Plissard, E. P. A. M. Bakkers, and L. P. Kouwenhoven, Science 336, 1003 (2012).

[19] M. T. Deng, C. L. Yu, G. Y. Huang, M. Larsson, P. Caroff, and H. Q. Xu, Nano Lett. 12, 6414 (2012).

[20] A. Das, Y. Ronen, Y. Most, Y. Oreg, M. Heiblum, and H. Shtrikman, Nat. Phys. 8, 887 (2012).

[21] L. P. Rokhinson, X. Liu, and J. K. Furdyna, Nat. Phys. 8, 795 (2012).

[22] A. D. K. Finck, D. J. Van Harlingen, P. K. Mohseni, K. Jung, and X. Li, Phys. Rev. Lett. 110, 126406 (2013).

[23] M. Leijnse and K. Flensberg, Phys. Rev. Lett. 107, 210502 (2011).

[24] M. Leijnse and K. Flensberg, Phys. Rev. B 86, 104511 (2012). 
[25] S. Tewari, C. Zhang, S. Das Sarma, C. Nayak, and D. H. Lee, Phys. Rev. Lett. 100, 027001 (2008).

[26] D. E. Liu and H. U. Baranger, Phys. Rev. B 84, 201308 (2011).

[27] M. Leijnse and K. Flensberg, Phys. Rev. B 84, 140501(R) (2011).

[28] Z. Wang, X. Y. Hu, Q. F. Liang, and X. Hu, Phys. Rev. B 87, 214513 (2013).

[29] Y. Cao, P. Wang, G. Xiong, M. Gong, and X. Q. Li, Phys. Rev. B 86, 115311 (2012).

[30] H. F. Lu, H. Z. Lu, and S. Q. Shen, Phys. Rev. B 86, 075318 (2012); 88, 159908 (2013).

[31] B. Zocher and B. Rosenow, Phys. Rev. Lett. 111, 036802 (2013).

[32] J. Liu, F. C. Zhang, and K. T. Law, Phys. Rev. B 88, 064509 (2013).

[33] P. Wang, Y. Cao, M. Gong, S. S. Li, and X. Q. Li, Phys. Lett. A 378, 937 (2014).

[34] P. Wang, Y. Cao, M. Gong, G. Xiong, and X. Q. Li, Europhys. Lett. 103, 57016 (2013).

[35] J. D. Sau and S. Das Sarma, Nat. Commun. 3, 964 (2012).

[36] C. J. Bolech and E. Demler, Phys. Rev. Lett. 98, 237002 (2007).

[37] K. T. Law, P. A. Lee, and T. K. Ng, Phys. Rev. Lett. 103, 237001 (2009).

[38] J. Nilsson, A. R. Akhmerov, and C. W. J. Beenakker, Phys. Rev. Lett. 101, 120403 (2008).

[39] B. H. Wu and J. C. Cao, Phys. Rev. B 85, 085415 (2012).

[40] A. Komnik, Phys. Rev. B 79, 245102 (2009).

[41] K. Joho, S. Maier, and A. Komnik, Phys. Rev. B 86, 155304 (2012).

[42] Y. Meir and N. S. Wingreen, Phys. Rev. Lett. 68, 2512 (1992).

[43] A. P. Jauho, N. S. Wingreen, and Y. Meir, Phys. Rev. B 50, 5528 (1994).

[44] Q. F. Sun, J. Wang, and H. Guo, Phys. Rev. B 71, 165310 (2005).

[45] N. Lambert, R. Aguado, and T. Brandes, Phys. Rev. B 75, 045340 (2007).

[46] V. Kashcheyevs, A. Aharony, and O. Entin-Wohlman, Phys. Rev. B 73, 125338 (2006).

[47] A. Mitra and A. J. Millis, Phys. Rev. B 76, 085342 (2007).

[48] C. Timm, Phys. Rev. B 77, 195416 (2008).
[49] C. Pöltl, C. Emary, and T. Brandes, Phys. Rev. B 80, 115313 (2009).

[50] H. Z. Lu, B. Zhou, and S. Q. Shen, Phys. Rev. B 79, 174419 (2009).

[51] S. Das Sarma, J. D. Sau, and T. D. Stanescu, Phys. Rev. B 86, 220506 (2012).

[52] D. T. McClure, L. DiCarlo, Y. Zhang, H. A. Engel, C. M. Marcus, M. P. Hanson, and A. C. Gossard, Phys. Rev. Lett. 98, 056801 (2007).

[53] Y. Zhang, L. DiCarlo, D. T. McClure, M. Yamamoto, S. Tarucha, C. M. Marcus, M. P. Hanson, and A. C. Gossard, Phys. Rev. Lett. 99, 036603 (2007).

[54] L. Hofstetter, S. Csonka, J. Nygård, and C. Schönenberger, Nature (London) 461, 960 (2009).

[55] L. Hofstetter, S. Csonka, A. Baumgartner, G. Fülöp, S d'Hollosy, J. Nygård, and C. Schönenberger, Phys. Rev. Lett. 107, 136801 (2011).

[56] J. Wei and V. Chandrasekhar, Nat. Phys. 6, 494 (2010).

[57] A. Das, Y. Ronen, M. Heiblum, D. Mahalu, A. V. Kretinin, and H. Shtrikman, Nat. Commun. 3, 1165 (2012).

[58] J. Eldridge, M. G. Pala, M. Governale, and J. König, Phys. Rev. B 82, 184507 (2010).

[59] M. Governale, M. G. Pala, and J. König, Phys. Rev. B 77, 134513 (2008).

[60] H. Bruus and K. Flensberg, Many-Body Quantum Theory in Condensed Matter Physics (Oxford University Press, Oxford, UK, 2004).

[61] N. A. Zimbovskayaa and M. R. Pederson, Phys. Rep. 509, 1 (2011).

[62] L. S. Levitov, in Quantum Noise in Mesoscopic Physics, edited by Y. V. Nazarov (Kluwer, Dordrecht, 2003).

[63] Y. M. Blanter and M. Büttiker, Phys. Rep. 336, 1 (2000).

[64] L. D. Contreras-Pulido and R. Aguado, Phys. Rev. B 77, 155420 (2008).

[65] A. J. White, A. Migliore, M. Galperin, and A. Nitzan, J. Chem. Phys. 138, 174111 (2013).

[66] R. Volkovich, R. Hartle, M. Thoss, and U. Peskin, Phys. Chem. Chem. Phys. 13, 14333 (2011).

[67] T. F. Fang and S. J. Wang, J. Phys.: Condens. Matter 19, 026204 (2007). 